\title{
Clinically Significant Drug Interactions Between Psychotropic Agents and Repurposed COVID-19 Therapies
}

\author{
Milo Gatti ${ }^{1,2}$ (D) Fabrizio De Ponti ${ }^{1}$ (D) Federico Pea ${ }^{1,2}$ (D)
}

Accepted: 26 March 2021 / Published online: 18 April 2021

(c) The Author(s) 2021

\begin{abstract}
As many patients with underlying psychiatric disorders may be infected with COVID-19, and COVID-19-affected subjects may frequently experience a new onset of psychiatric manifestations, concomitant use of psychotropic medications and COVID-19 therapies is expected to be highly likely and raises concerns of clinically relevant drug interactions. In this setting, four major mechanisms responsible for drug interactions involving psychotropic agents and COVID-19 therapies may be identified: (1) pharmacokinetic drug-drug interactions mainly acting on cytochrome P450; (2) pharmacodynamic drug-drug interactions resulting in additive or synergistic toxicity; (3) drug-disease interactions according to stage and severity of the disease; and (4) pharmacogenetic issues associated with polymorphisms of cytochrome P450 isoenzymes. In this review, we summarise the available literature on relevant drug interactions between psychotropic agents and COVID-19 therapies, providing practical clinical recommendations and potential management strategies according to severity of illness and clinical scenario.
\end{abstract}

\section{Introduction}

The coronavirus disease 2019 (COVID-19) is a respiratory tract infection caused by severe acute respiratory syndrome coronavirus 2, first identified in the Wuhan State of China at the end of 2019 [1]. COVID-19 was stated as a public health emergency of international concern on 30 January, 2020, and subsequently listed as a global pandemic on 11 March, 2020 by the World Health Organization [2, 3]. As of 13 March, 2021, more than 117 million cases and 2.5 million deaths were reported in 220 countries [4]. COVID19 may be responsible for variable clinical manifestations, from an asymptomatic infection to severe respiratory distress syndrome associated with multi-organ failure [5].

Currently, the European Medicines Agency (EMA) and the US Food and Drug Administration (FDA) have recommended granting a marketing authorisation only to remdesivir [6, 7]. Specifically, remdesivir is approved only for adults and adolescents (aged $\geq 12$ years) with COVID-19

Federico Pea

federico.pea@unibo.it

1 Department of Medical and Surgical Sciences, Alma Mater Studiorum, University of Bologna, Via Massarenti, 9, 40138 Bologna, Italy

2 SSD Clinical Pharmacology, University Hospital IRCCS Policlinico Sant'Orsola, Bologna, Italy

\section{Key Points}

Clinically relevant drug interactions between psychotropic agents and COVID-19 agents may result from pharmacokinetic/pharmacodynamic properties of the involved medications or may be caused by a drug-disease impact of moderate-to-severe forms of COVID-19, leading to multi-organ failure and a cytokine storm.

Lopinavir/ritonavir and darunavir/cobicistat pose the major concern in terms of clinically relevant interactions with psychotropic agents. Concomitant administration of protease inhibitors with haloperidol, quetiapine, ziprasidone, carbamazepine, midazolam and triazolam should be avoided because of increased toxicity. A low risk of clinically relevant interactions is expected with other COVID-19 agents (corticosteroids, heparin, immunomodulators, anti-JAK and remdesivir).

Psychotropic agents exhibiting a favourable pharmacokinetic/pharmacodynamic profile (sertraline, vortioxetine, milnacipran, olanzapine, paliperidone, oxazepam-like benzodiazepines) should be preferred in patients with COVID-19 requiring protease inhibitors. 
pneumonia requiring supplemental oxygen. Furthermore, the FDA recently granted an Emergency Use Authorization to the investigational neutralising IgG1 monoclonal antibodies bamlanivimab and casirivimab/imdevimab for treating recently diagnosed, mild-to-moderate COVID19 infection in patients who are aged $\geq 12$ years $[8,9]$. However, off-label use of different agents (e.g. darunavir/ cobicistat, lopinavir/ritonavir, tocilizumab, siltuximab, ruxolitinib, canakinumab, heparin, corticosteroids, colchicine) is currently implemented in patients with COVID-19, although several concerns in terms of efficacy and safety have been raised, including the risk of clinically relevant interactions with different classes of drugs [10-13].

Considering that many patients with underlying psychiatric disorders may be infected with COVID-19, and that up to $25 \%$ of patients affected by COVID-19 were shown to experience a new onset of psychiatric manifestations (such as anxiety, depression, insomnia, obsessivecompulsive disorder, reactive psychosis or post-traumatic stress disorder) [14-19], concomitant use of psychotropic medications and COVID-19 therapies is highly probable. Additionally, intensive care support and COVID-19 therapies showing psychiatric side effects might be an additional risk factor for the onset of psychiatric symptoms and delirium, requiring adequate treatment with psychotropic agents [20]. Psychotropic medications may be especially involved in clinically relevant drug interactions in the COVID-19 scenario, by virtue of: (1) extensive activity as substrates and/or inhibitors/inducers of cytochrome P450 (CYP) isoenzymes, thus potentially causing bidirectional pharmacokinetic (PK) interactions with COVID-19 therapies; (2) synergistic toxicity with different COVID19 agents, leading to clinically relevant pharmacodynamic (PD) interactions (e.g. QT prolongation); (3) the relevant impact of COVID-19 in causing multiple organ failure (e.g. liver and renal impairment, haematological effects), and a cytokine storm relevantly affecting both PK/PD properties of psychotropic medications and CYP activity, thus resulting in significant drug-disease interactions [20-24]. Cytokine release syndrome represents a life-threatening systemic inflammatory syndrome involving elevated levels of circulating cytokines and immunecell hyperactivation, and may play a major role in causing multi-organ failure in the late stages of COVID-19 [24]. The elevated levels of circulating cytokines are responsible for both the impaired activity of different CYP450 isozymes and the use of several anti-inflammatory and/ or immunomodulatory agents in severe forms of COVID19 , thus contributing to the occurrence of drug-drug and drug-disease interactions.

Notably, a close relationship between the severity of COVID-19, clinical scenarios (from outpatient to intensive care unit [ICU] admission), and the use of specific psychotropic and COVID-19 therapies may be identified (Fig. 1). Antiviral agents are considered more appropriate in the early phase of infection, characterised by viral replication, while immunomodulatory agents should be reserved for later stages, characterised mainly by systemic hyperinflammation [5]. Likewise, while long-term psychiatric therapies are usually maintained in outpatient/inpatient settings in cases of mild-to-moderate COVID-19 infection, these medications are commonly discontinued in cases of severe COVID-19 forms requiring ICU admission [25]. In these latter cases, psychotropic use is limited to a few agents for sedation and treatment of delirium. Consequently, clinically relevant interactions between psychotropic agents and COVID-19 therapies, although demonstrated in healthy volunteers or predicted according to PK/PD properties, may not occur considering that the two agents are not concomitantly used in a specific scenario.

Although different reviews concerning drug interactions between psychotropic medications and COVID-19 therapies have just been published [20-22], data focusing on clinically relevant interactions in different COVID-19 scenarios are limited. This review aims to summarise the available literature on relevant drug interactions between psychotropic agents and COVID-19 therapies, providing practical clinical recommendations and potential management strategies according to severity of illness and clinical scenario.

\section{Search Strategy}

A literature search was conducted on PubMed-MEDLINE (until 7 January, 2021) to retrieve PK and PD features of currently used COVID-19 therapies and psychotropic agents, specifically involved in the occurrence of relevant drug-drug interactions (DDIs) and drug-disease interactions, namely: (1) activity on CYP and other transporters (e.g. P-glycoprotein [P-gp]); (2) QT prolongation; and (3) significant variations affecting PK/PD properties of selected agents according to the severity of COVID-19 (e.g. organ failure, cytokine storm). Only studies conducted in humans and published in English were included.

Data concerning the activity on CYP450 and on transporters of psychotropic medications and COVID-19 agents were retrieved from the summary of product characteristics and the review of Smolders et al. [26]. Information on drug-induced QT prolongation were extracted from Crediblemeds.org [27]. QT drugs were classified respectively as agents with known risk (substantial evidence supports the conclusion that these drugs prolong QT intervals and have a risk of torsades de pointes when used as directed in labelling), possible risk (substantial evidence supports the conclusion that these drugs can cause QT prolongation but there is insufficient evidence that the drugs, when used 


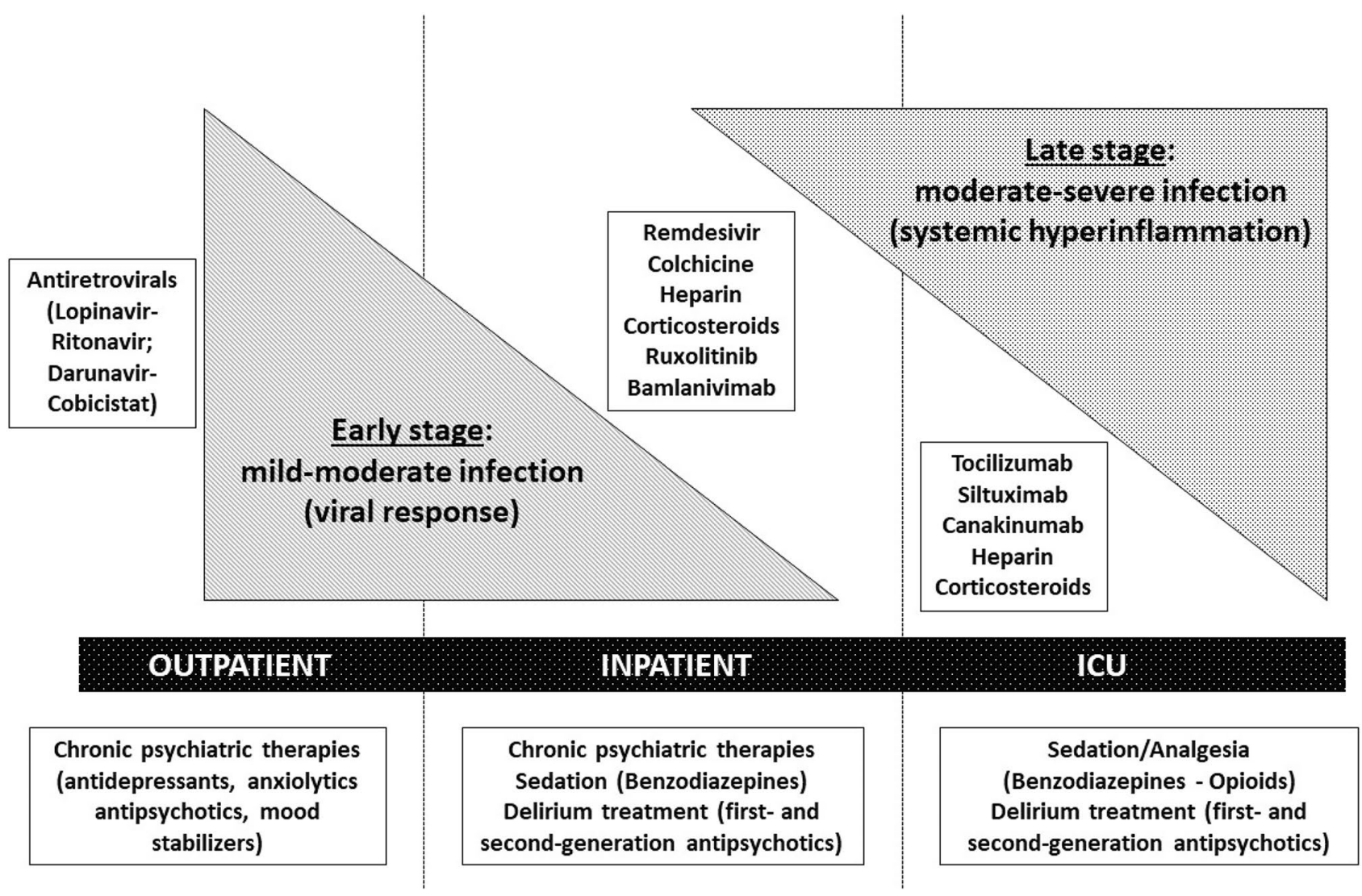

Fig. 1 Relationship between severity of COVID-19 disease, clinical scenarios, and use of specific psychotropic and COVID-19 therapies. ICU intensive care unit

as directed in labelling, have a risk of causing torsades de pointes) or conditional risk (substantial evidence supports the conclusion that these drugs prolong QT and have a risk of developing torsades de pointes but only under certain known conditions) [27].

Agents with a well-established use or under investigation for COVID-19 treatment were included in the analysis, namely: bamlanivimab, canakinumab, casirivimab/ imdevimab, colchicine, darunavir/cobicistat, dexamethasone, enoxaparin, lopinavir/ritonavir, remdesivir, ruxolitinib, siltuximab, tocilizumab. The following classes of psychotropic medications were investigated: antidepressants (agomelatine, amitriptyline, bupropion, citalopram, clomipramine, desipramine, doxepin, duloxetine, escitalopram, fluoxetine, fluvoxamine, imipramine, mianserin, milnacipran, mirtazapine, nortriptyline, paroxetine, phenelzine, reboxetine, sertraline, tranylcypromine, trazodone, venlafaxine, vortioxetine), antipsychotic agents (amisulpiride, aripiprazole, chlorpromazine, clozapine, fluphenazine, haloperidol, iloperidone, lurasidone, olanzapine, paliperidone, perphenazine, pimozide, quetiapine, risperidone, sertindole, sulpiride, thioridazine, ziprasidone, zuclopenthixol), mood stabilisers (carbamazepine, lamotrigine, lithium, valproic acid) and sedative hypnotics (alprazolam, bromazepam, brotizolam, chlordiazepoxide, clobazam, clonazepam, clorazepate, diazepam, flurazepam, lorazepam, lormetazepam, midazolam, oxazepam, temazepam, triazolam, zolpidem, zopiclone).

A two-step approach was performed to detect clinically relevant drug interactions between psychotropic medications and COVID-19 agents. First, terms identifying psychotropic medications were combined with terms identifying COVID19 therapies, to retrieve PK studies or case reports/series assessing the clinical relevance of the interaction according to the guideline provided by the EMA [28]. All studies or case series/reports providing PK data or describing clinically relevant PD events resulting from the coadministration of the two specific agents were included in our review. Second, information on drug-drug PK and PD interactions between psychotropic medications and COVID-19 agents was searched by checking the updated COVID-19 drug interaction webpage of the University of Liverpool [29] and the summaries of product characteristics. Drug-drug interactions were classified into three different categories according to the degree of severity and clinical relevance: (1) low risk, identifying agents exhibiting no predicted interaction; 
(2) moderate risk, identifying agents exhibiting a predicted interaction according to PK/PD properties with unknown clinical relevance; (3) high risk, identifying agents exhibiting a clinically relevant interaction, for which avoidance of concomitant administration or dose adjustment should be considered. Finally, applicability in different COVID-19 scenarios was provided for each identified drug interaction, according to the severity of disease.

\section{Major Mechanisms Responsible for Drug Interactions Involving Psychotropic Medications in the COVID-19 Era}

Four major mechanisms responsible for drug interactions involving psychotropic agents and COVID-19 therapies may be identified: (1) PK interactions resulting in significant changes in disposition of psychotropic drugs or COVID-19 agents; (2) PD interactions resulting in additive or synergistic toxicity; (3) drug-disease interactions related to multiorgan failure and a cytokine storm commonly reported in moderate-to-severe COVID-19; and (4) pharmacogenetic issues associated with polymorphisms in CYP isoenzymes involved in the metabolism of both psychotropic and COVID-19 agents [20-24].

\subsection{PK Interactions}

Although drug interactions between psychotropic medications and COVID-19 therapies may theoretically involve each of the four PK ADME (absorption, distribution, metabolism, and elimination) phases [30], the most relevant mechanisms concern metabolic activity on the CYP microsomal system and drug transporters (mainly P-gp) according to lipophilic properties of psychotropic agents [26, 31, 32]. The influences of psychotropic medications and of COVID19 therapies on CYP450 and drug transporters activities are summarised in Tables 1 and 2, respectively. Psychotropic agents are largely metabolised by the CYP system. The CYP2D6 and CYP2C19 isozymes are mainly involved in the metabolism of several antidepressants (including selective serotonin reuptake inhibitors [SSRIs] and tricyclic antidepressants [TCAs]) and antipsychotic medications (of both first and second generation), while different sedative hypnotics and mood stabilisers (apart from oxazepam-like benzodiazepines, valproic acid, lamotrigine and lithium) are metabolised by the CYP3A4 isozyme [26]. Additionally, concerns in terms of relevant drug interactions may arise as a result of inhibitory or induced activity on CYP. Fluoxetine, paroxetine and bupropion are strong inhibitors of CYP2D6, fluvoxamine is a strong inhibitor of both CYP1A2 and $\mathrm{CYP} 2 \mathrm{C} 19$, whereas carbamazepine is a strong inducer of both CYP3A4 and P-gp [26]. With regard to COVID-19 therapies, the major issues in terms of relevant drug interactions may be associated with the use of antiretrovirals. Both darunavir/cobicistat and lopinavir/ritonavir are strong inhibitors of both CYP3A4 and CYP2D6. Colchicine and ruxolitinib are largely metabolised by CYP3A4, and thus their metabolism may be significantly affected in the case of coadministration with psychotropic medications that act as inhibitors or inducers of this isoenzyme.

\subsection{PD Interactions}

Clinicians should be aware that PD interactions may result in clinically relevant outcomes, triggered by additive or synergistic toxicity when certain psychotropic medications and COVID-19 therapies are concomitantly administered [10]. In this regard, several antidepressants and antipsychotic agents exhibit a well-established risk of QT prolongation (Table 1). The concomitant use of lopinavir/ritonavir may increase this risk by virtue of the PK/PD properties of this antiviral combination (Table 2), potentially leading to fatal arrhythmias [33]. Expected toxicity is commonly additive, extremely rarely synergistic. In the COVID-19 scenario, the main concerns involving PD interactions may be represented by the risk of QT prolongation and possible consequent fatal arrhythmias, coupled with additive haematological toxicity in patients co-treated with clozapine and ruxolitinib and/or anti-interleukin (IL)-1/6 agents.

\subsection{Drug-Disease Interactions}

The degree of severity of COVID-19 infection may be responsible for significant variations in the disposition of several psychotropic agents due to multi-organ failure and a cytokine storm commonly reported in moderate-to-severe forms of COVID-19 [34]. Specifically, the increased level of pro-inflammatory cytokines (e.g. IL-1 and IL-6) may consistently reduce the activity of CYP3A4, CYP1A2 and CYP2C19 [23, 35-37], and this may potentially affect the metabolism of those psychotropic agents that are substrates of these isoenzymes. Interestingly, among patients with severe COVID-19 treated with darunavir, a CYP3A4 substrate, it was shown that high IL-6 levels significantly decrease drug clearance [38]. This supports the idea that in severe forms of COVID-19, dosing optimisation of CYP3A4 substrates, including several psychotropic medications, may be needed. Conversely, no significant influence of proinflammatory cytokine levels was documented on CYP2D6 activity $[23,37]$. This suggests that the metabolism of several antidepressants and antipsychotic agents that are CYP2D6 substrates should not be affected by the hyperimmune response observed in severe forms of COVID-19.

A cytokine storm usually occurs in patients with severe COVID-19 who may often need ICU admission [24]. In this 
Table 1 Summary of the activity of the different psychotropic agents on CYP450. Activity on transporters and relevant PD effects (including QT prolongation) are also indicated

\begin{tabular}{|c|c|c|c|c|c|c|c|}
\hline Agents & CYP1A2 & CYP2C $8 / 9$ & CYP2C19 & CYP2D6 & CYP3A4/5 & $\begin{array}{l}\text { Glucuronidation } \\
\text { transporters }\end{array}$ & QT prolongation \\
\hline \multicolumn{8}{|l|}{ Antidepressants } \\
\hline \multicolumn{8}{|l|}{ SSRIs } \\
\hline Citalopram & Inhibitor (weak) & - & $\begin{array}{l}\text { Substrate } \\
\text { Inhibitor (weak) }\end{array}$ & $\begin{array}{l}\text { Substrate } \\
\text { (minor) } \\
\text { Inhibitor (weak) }\end{array}$ & Substrate & - & Known risk \\
\hline Escitalopram & - & - & Substrate & Inhibitor (weak) & Substrate & - & Known risk \\
\hline Fluoxetine & $\begin{array}{l}\text { Substrate } \\
\text { (minor) } \\
\text { Inhibitor (weak) }\end{array}$ & $\begin{array}{l}\text { Substrate } \\
\text { Inhibitor (weak) }\end{array}$ & $\begin{array}{l}\text { Substrate } \\
\text { (minor) } \\
\text { Inhibitor (mod- } \\
\text { erate) }\end{array}$ & $\begin{array}{l}\text { Substrate } \\
\text { Inhibitor } \\
\text { (strong) }\end{array}$ & $\begin{array}{r}\text { Substrate } \\
\text { (minor) }\end{array}$ & - & Conditional risk \\
\hline Fluvoxamine & $\begin{array}{l}\text { Substrate } \\
\text { Inhibitor } \\
\text { (strong) }\end{array}$ & Inhibitor (weak) & $\begin{array}{l}\text { Inhibitor } \\
\text { (strong) }\end{array}$ & $\begin{array}{l}\text { Substrate } \\
\text { Inhibitor (weak) }\end{array}$ & Inhibitor (weak) & - & Conditional risk \\
\hline Paroxetine & Inhibitor (weak) & Inhibitor (weak) & Inhibitor (weak) & $\begin{array}{l}\text { Substrate } \\
\text { Inhibitor } \\
\text { (strong) }\end{array}$ & - & - & Conditional risk \\
\hline Sertraline & Inhibitor (weak) & $\begin{array}{l}\text { Substrate } \\
\text { (minor) } \\
\text { Inhibitor (weak) }\end{array}$ & $\begin{array}{l}\text { Substrate } \\
\text { (minor) } \\
\text { Inhibitor (mod- } \\
\text { erate) }\end{array}$ & $\begin{array}{l}\text { Substrate } \\
\text { (minor) } \\
\text { Inhibitor (mod- } \\
\text { erate) }\end{array}$ & $\begin{array}{r}\text { Substrate } \\
\text { (minor) }\end{array}$ & - & Conditional risk \\
\hline Vortioxetine & - & $\begin{array}{r}\text { Substrate } \\
\text { (minor) }\end{array}$ & $\begin{array}{r}\text { Substrate } \\
\text { (minor) }\end{array}$ & Substrate & Substrate & $\begin{array}{l}\text { P-gp: } \\
\text { Substrate } \\
\text { (minor) } \\
\text { Inhibitor (weak) }\end{array}$ & - \\
\hline \multicolumn{8}{|l|}{ SNRIs } \\
\hline $\begin{array}{l}\text { Desvenlafax- } \\
\text { ine }\end{array}$ & - & - & - & - & $\begin{array}{l}\text { Substrate } \\
\text { (minor) }\end{array}$ & UGT substrate & - \\
\hline Duloxetine & Substrate & - & - & $\begin{array}{l}\text { Substrate } \\
\text { Inhibitor (mod- } \\
\text { erate) }\end{array}$ & - & - & - \\
\hline Reboxetine & - & - & - & Inhibitor (weak) & $\begin{array}{l}\text { Substrate } \\
\text { Inhibitor (weak) }\end{array}$ & - & - \\
\hline Venlafaxine & - & $\begin{array}{r}\text { Substrate } \\
\text { (minor) }\end{array}$ & $\begin{array}{r}\text { Substrate } \\
\text { (minor) }\end{array}$ & $\begin{array}{l}\text { Substrate } \\
\text { Inhibitor (weak) }\end{array}$ & $\begin{array}{l}\text { Substrate } \\
\text { Inhibitor (weak) }\end{array}$ & - & Possible risk \\
\hline \multicolumn{8}{|l|}{ TCAs } \\
\hline Amitriptyline & $\begin{array}{l}\text { Substrate } \\
\text { (minor) } \\
\text { Inhibitor (weak) }\end{array}$ & $\begin{array}{l}\text { Substrate } \\
\text { (minor) } \\
\text { Inhibitor (weak) }\end{array}$ & $\begin{array}{l}\text { Substrate } \\
\text { (minor) } \\
\text { Inhibitor (weak) }\end{array}$ & $\begin{array}{l}\text { Substrate } \\
\text { Inhibitor (weak) }\end{array}$ & $\begin{array}{r}\text { Substrate } \\
\text { (minor) }\end{array}$ & Substrate (P-gp) & Conditional risk \\
\hline Clomipramine & Substrate & - & Substrate & $\begin{array}{l}\text { Substrate } \\
\text { Inhibitor (mod- } \\
\text { erate) }\end{array}$ & $\begin{array}{r}\text { Substrate } \\
\text { (minor) }\end{array}$ & - & Conditional risk \\
\hline Doxepin & $\begin{array}{r}\text { Substrate } \\
\text { (minor) }\end{array}$ & - & $\begin{array}{r}\text { Substrate } \\
\text { (minor) }\end{array}$ & Substrate & $\begin{array}{r}\text { Substrate } \\
\text { (minor) }\end{array}$ & - & Conditional risk \\
\hline Imipramine & $\begin{array}{l}\text { Substrate } \\
\text { (minor) } \\
\text { Inhibitor (weak) }\end{array}$ & - & $\begin{array}{l}\text { Substrate } \\
\text { Inhibitor (weak) }\end{array}$ & $\begin{array}{l}\text { Substrate } \\
\text { Inhibitor (mod- } \\
\text { erate) }\end{array}$ & $\begin{array}{r}\text { Substrate } \\
\text { (minor) }\end{array}$ & - & Possible risk \\
\hline Nortriptyline & $\begin{array}{r}\text { Substrate } \\
\text { (minor) }\end{array}$ & - & $\begin{array}{r}\text { Substrate } \\
\text { (minor) }\end{array}$ & $\begin{array}{l}\text { Substrate } \\
\text { Inhibitor (weak) }\end{array}$ & $\begin{array}{r}\text { Substrate } \\
\text { (minor) }\end{array}$ & Substrate (P-gp) & Possible risk \\
\hline \multicolumn{8}{|l|}{$M A O-I$} \\
\hline Phenelzine & - & - & - & - & - & - & - \\
\hline $\begin{array}{l}\text { Tranylcy- } \\
\text { promine }\end{array}$ & - & - & - & - & - & - & - \\
\hline \multicolumn{8}{|l|}{ Others } \\
\hline Agomelatine & Substrate & Substrate & Substrate & - & - & - & - \\
\hline
\end{tabular}


Table 1 (continued)

\begin{tabular}{|c|c|c|c|c|c|c|c|}
\hline Agents & CYP1A2 & CYP2C8/9 & CYP2C19 & CYP2D6 & CYP3A4/5 & $\begin{array}{l}\text { Glucuronidation } \\
\text { transporters }\end{array}$ & QT prolongation \\
\hline Bupropion & $\begin{array}{r}\text { Substrate } \\
\text { (minor) }\end{array}$ & $\begin{array}{r}\text { Substrate } \\
\text { (minor) }\end{array}$ & - & $\begin{array}{l}\text { Substrate } \\
\text { (minor) } \\
\text { Inhibitor } \\
\text { (strong) }\end{array}$ & $\begin{array}{r}\text { Substrate } \\
\text { (minor) }\end{array}$ & $\begin{array}{l}\text { Inhibitor } \\
\text { (OCT2) }\end{array}$ & - \\
\hline Mianserin & - & - & - & Substrate & - & - & Possible risk \\
\hline Milnacipran & - & - & - & - & - & UGT substrate & - \\
\hline Mirtazapine & $\begin{array}{l}\text { Substrate } \\
\text { Inhibitor (weak) }\end{array}$ & $\begin{array}{r}\text { Substrate } \\
\text { (minor) }\end{array}$ & - & Substrate & Substrate & - & Possible risk \\
\hline Trazodone & - & - & - & $\begin{array}{r}\text { Substrate } \\
\text { (minor) }\end{array}$ & Substrate & Inducer (P-gp) & Conditional risk \\
\hline \multicolumn{8}{|c|}{ Antipsychotic agents } \\
\hline \multicolumn{8}{|l|}{ First-generation } \\
\hline Amisulpiride & - & - & - & - & - & - & Conditional risk \\
\hline $\begin{array}{l}\text { Chlorproma- } \\
\text { zine }\end{array}$ & $\begin{array}{r}\text { Substrate } \\
\text { (minor) }\end{array}$ & - & - & Substrate & $\begin{array}{r}\text { Substrate } \\
\text { (minor) }\end{array}$ & - & Known risk \\
\hline Fluphenazine & $\begin{array}{l}\text { Substrate } \\
\text { Inhibitor (weak) }\end{array}$ & Inhibitor (weak) & - & $\begin{array}{l}\text { Substrate } \\
\text { Inhibitor (weak) }\end{array}$ & - & - & - \\
\hline Haloperidol & $\begin{array}{r}\text { Substrate } \\
\text { (minor) }\end{array}$ & - & - & $\begin{array}{l}\text { Substrate } \\
\text { Inhibitor (mod- } \\
\text { erate) }\end{array}$ & Substrate & - & Known risk \\
\hline Perphenazine & $\begin{array}{l}\text { Substrate } \\
\text { (minor) } \\
\text { Inhibitor (weak) }\end{array}$ & $\begin{array}{r}\text { Substrate } \\
\text { (minor) }\end{array}$ & $\begin{array}{r}\text { Substrate } \\
\text { (minor) }\end{array}$ & $\begin{array}{l}\text { Substrate } \\
\text { Inhibitor (weak) }\end{array}$ & $\begin{array}{r}\text { Substrate } \\
\text { (minor) }\end{array}$ & - & Possible risk \\
\hline Pimozide & Substrate & - & Inhibitor (weak) & $\begin{array}{l}\text { Substrate } \\
\text { Inhibitor (weak) }\end{array}$ & Substrate & - & Known risk \\
\hline Sulpiride & - & - & - & - & - & - & Known risk \\
\hline Thioridazine & - & - & - & Substrate & $\begin{array}{l}\text { Substrate } \\
\text { (minor) } \\
\text { Inducer } \\
\text { (moderate) }\end{array}$ & - & Known risk \\
\hline \multicolumn{8}{|c|}{ Second-generation } \\
\hline Aripiprazole & - & - & - & Substrate & Substrate & - & Possible risk \\
\hline Cariprazine & - & - & - & $\begin{array}{l}\text { Substrate } \\
\text { (minor) }\end{array}$ & Substrate & - & - \\
\hline Clozapine & $\begin{array}{l}\text { Substrate } \\
\text { Inhibitor (weak) }\end{array}$ & $\begin{array}{l}\text { Substrate } \\
\text { (minor) } \\
\text { Inhibitor (weak) }\end{array}$ & $\begin{array}{l}\text { Substrate } \\
\text { (minor) } \\
\text { Inhibitor (weak) }\end{array}$ & $\begin{array}{l}\text { Substrate } \\
\text { (minor) } \\
\text { Inhibitor (mod- } \\
\text { erate) }\end{array}$ & $\begin{array}{l}\text { Substrate } \\
\text { (minor) } \\
\text { Inhibitor (weak) }\end{array}$ & - & Possible risk \\
\hline Iloperidone & - & - & - & Substrate & Substrate & - & Possible risk \\
\hline Lurasidone & - & - & - & - & $\begin{array}{l}\text { Substrate } \\
\text { Inhibitor (weak) }\end{array}$ & Substrate (P-gp) & Possible risk \\
\hline Olanzapine & $\begin{array}{l}\text { Substrate } \\
\text { Inhibitor (weak) }\end{array}$ & Inhibitor (weak) & Inhibitor (weak) & $\begin{array}{l}\text { Substrate } \\
\text { (minor) } \\
\text { Inhibitor (weak) }\end{array}$ & Inhibitor (weak) & UGT substrate & Conditional risk \\
\hline Paliperidone & - & - & - & - & - & $\begin{array}{l}\text { Inhibitor } \\
\text { (weak; P-gp) }\end{array}$ & Possible risk \\
\hline Quetiapine & - & - & - & $\begin{array}{r}\text { Substrate } \\
\text { (minor) }\end{array}$ & Substrate & - & Conditional risk \\
\hline Risperidone & - & - & - & $\begin{array}{l}\text { Substrate } \\
\text { Inhibitor (weak) }\end{array}$ & $\begin{array}{r}\text { Substrate } \\
\text { (minor) }\end{array}$ & Substrate (P-gp) & Conditional risk \\
\hline Sertindole & - & - & - & Substrate & Substrate & - & Known risk \\
\hline Ziprasidone & - & - & - & - & $\begin{array}{r}\text { Substrate } \\
\text { (minor) }\end{array}$ & - & Conditional risk \\
\hline
\end{tabular}


Table 1 (continued)

\begin{tabular}{|c|c|c|c|c|c|c|c|}
\hline Agents & CYP1A2 & CYP2C8/9 & CYP2C19 & CYP2D6 & CYP3A4/5 & $\begin{array}{l}\text { Glucuronidation } \\
\text { transporters }\end{array}$ & QT prolongation \\
\hline $\begin{array}{l}\text { Zuclopen- } \\
\text { thixol }\end{array}$ & - & - & - & Substrate & $\begin{array}{r}\text { Substrate } \\
\text { (minor) }\end{array}$ & - & Possible risk \\
\hline \multicolumn{8}{|l|}{ Mood stabilisers } \\
\hline $\begin{array}{l}\text { Carbamaz- } \\
\text { epine }\end{array}$ & - & $\begin{array}{r}\text { Substrate } \\
\text { (minor) }\end{array}$ & - & - & $\begin{array}{l}\text { Substrate } \\
\text { Inducer (strong) }\end{array}$ & $\begin{array}{l}\text { Substrate (P-gp) } \\
\text { Inducer } \\
\text { (strong; P-gp) }\end{array}$ & - \\
\hline Lamotrigine & - & - & - & - & - & UGT substrate & - \\
\hline Lithium & - & - & - & - & - & - & Possible risk \\
\hline Valproic acid & - & - & - & - & $\begin{array}{l}- \\
\text { Inhibitor } \\
\quad \text { (strong) }\end{array}$ & UGT substrate & - \\
\hline \multicolumn{8}{|l|}{ Sedative hypnotics } \\
\hline Alprazolam & - & - & - & - & $\begin{array}{l}\text { Substrate } \\
\text { Inhibitor (weak) }\end{array}$ & - & - \\
\hline Bromazepam & Substrate & - & - & - & $\begin{array}{r}\text { Substrate } \\
(\text { minor })\end{array}$ & - & - \\
\hline Brotizolam & - & - & - & - & Substrate & - & - \\
\hline $\begin{array}{l}\text { Chlordiaz- } \\
\text { epoxide }\end{array}$ & - & - & - & - & Substrate & - & - \\
\hline Clobazam & - & - & $\begin{array}{l}\text { Substrate } \\
\text { Inhibitor (weak) }\end{array}$ & $\begin{array}{l}\text { Inhibitor (mod- } \\
\text { erate) }\end{array}$ & $\begin{array}{l}\text { Substrate } \\
\quad \text { (minor) } \\
\text { Inducer (weak) }\end{array}$ & $\begin{array}{l}\text { Substrate (P- } \\
\text { gp) }\end{array}$ & - \\
\hline Clonazepam & - & - & - & - & Substrate & - & - \\
\hline Clorazepate & - & - & - & - & Substrate & - & - \\
\hline Diazepam & $\begin{array}{r}\text { Substrate } \\
\text { (minor) }\end{array}$ & $\begin{array}{l}\text { Substrate } \\
\text { (minor) }\end{array}$ & $\begin{array}{l}\text { Substrate } \\
\text { Inhibitor (weak) }\end{array}$ & - & $\begin{array}{l}\text { Substrate } \\
\text { Inhibitor }\end{array}$ & - & - \\
\hline Eszopiclone & - & - & - & - & Substrate & - & - \\
\hline Flurazepam & - & - & - & - & Substrate & - & - \\
\hline Lorazepam & - & - & - & - & - & UGT substrate & - \\
\hline Lormetazepam & - & - & - & - & - & UGT substrate & - \\
\hline Midazolam & - & Inhibitor (weak) & - & - & $\begin{array}{l}\text { Substrate } \\
\text { Inhibitor (weak) }\end{array}$ & - & - \\
\hline Oxazepam & - & - & - & - & - & UGT substrate & - \\
\hline Temazepam & - & $\begin{array}{r}\text { Substrate } \\
\text { (minor) }\end{array}$ & $\begin{array}{r}\text { Substrate } \\
\text { (minor) }\end{array}$ & - & $\begin{array}{r}\text { Substrate } \\
\text { (minor) }\end{array}$ & UGT substrate & - \\
\hline Triazolam & - & - & - & - & Substrate & - & - \\
\hline Zolpidem & $\begin{array}{r}\text { Substrate } \\
\text { (minor) }\end{array}$ & - & $\begin{array}{r}\text { Substrate } \\
\text { (minor) }\end{array}$ & $\begin{array}{r}\text { Substrate } \\
\text { (minor) }\end{array}$ & Substrate & - & - \\
\hline Zopiclone & - & $\begin{array}{r}\text { Substrate } \\
\text { (minor) }\end{array}$ & - & - & Substrate & - & - \\
\hline
\end{tabular}

Data are retrieved from Smolders et al. [26] and the summary of product characteristics of the different agents for activity on CYP450 and other transporters, while data for QT prolongation were retrieved from Crediblemeds.org

$C Y P$ cytochrome $\mathrm{P} 450, O C T 2$ organic cation transporter 2, MAO-I monoamine oxidase inhibitors, $P D$ pharmacodynamic, $P$-g $p$ P-glycoprotein, TCAs tricyclic antidepressants, UGT UDP-glucuronosyltransferase, SNRIs serotonin-noradrenaline reuptake inhibitors, SSRIs selective serotonin reuptake inhibitors, - indicates no activity

setting, a simplification of a long-term medication regime is commonly performed, including the abrupt discontinuation of oral psychotropic agents [39]. This may significantly lower the risk of DDIs that is generally restricted only to the use of parenteral agents for sedation and treatment of delirium (e.g. midazolam, haloperidol). However, some patients may be receiving long-acting injectable antipsychotic agents to manage schizophrenia or bipolar disorder. In this scenario, clinically relevant drug interactions may occur despite ICU admission because of the long elimination half-life of the agents administered in these formulations. 


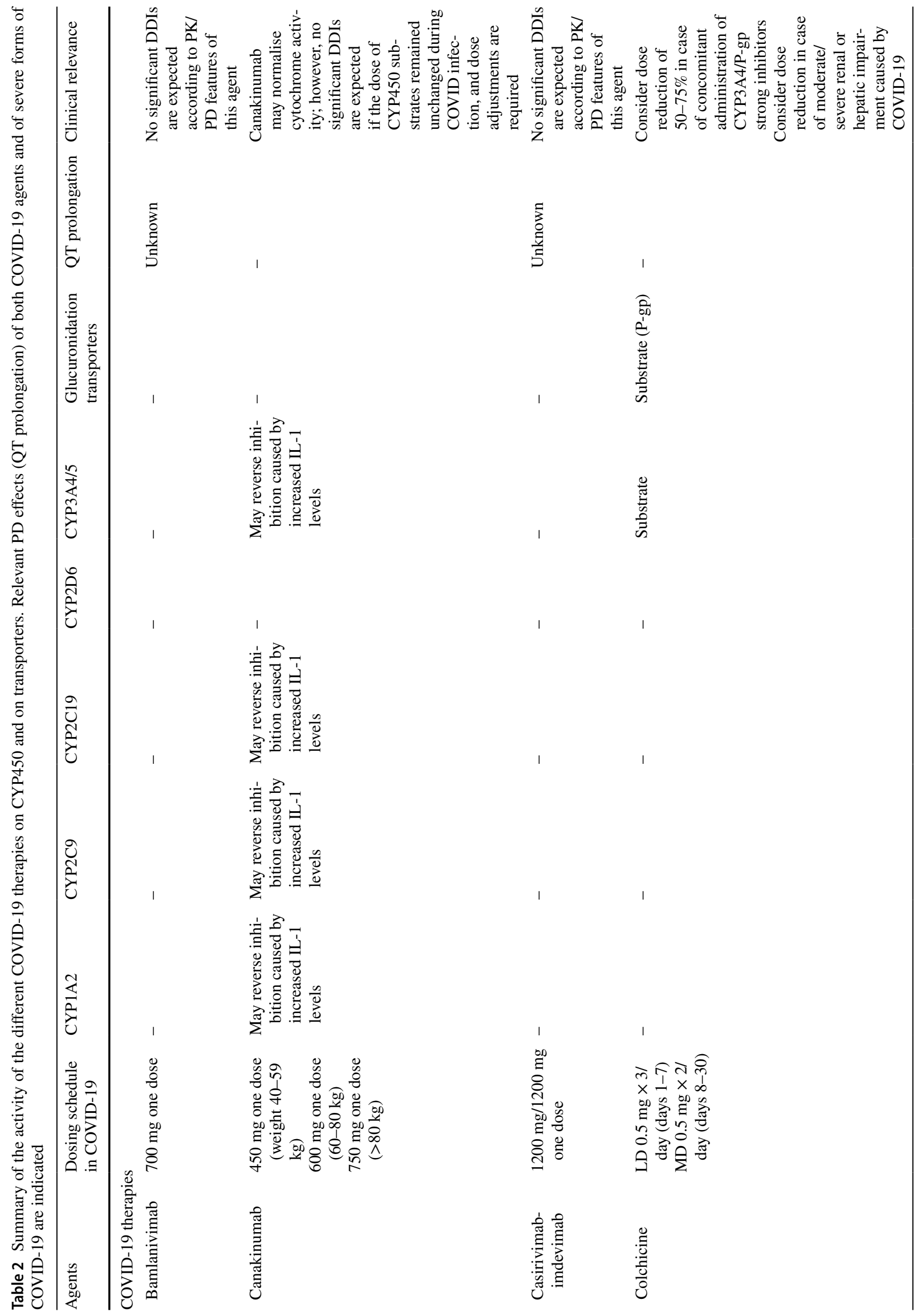




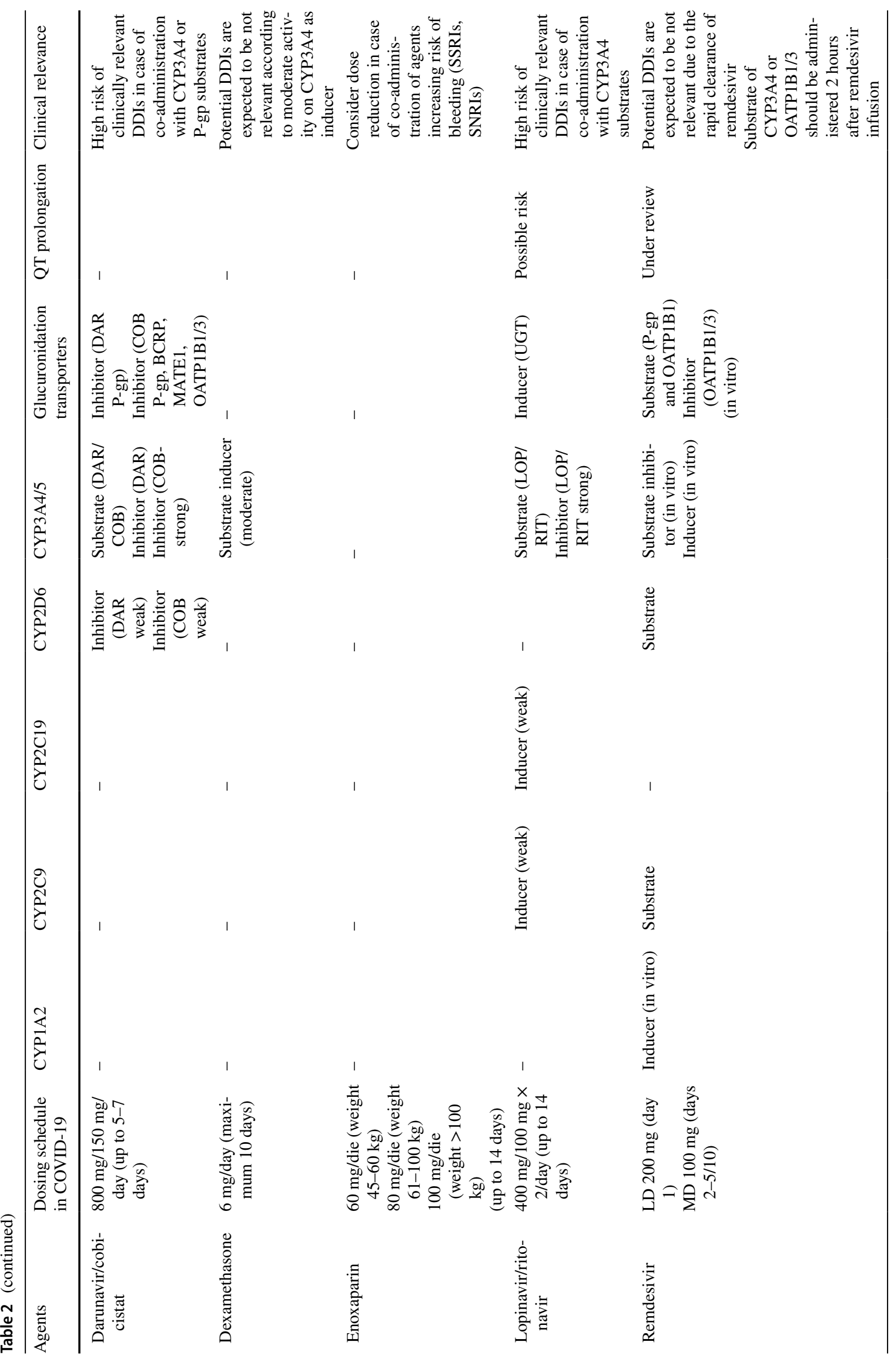




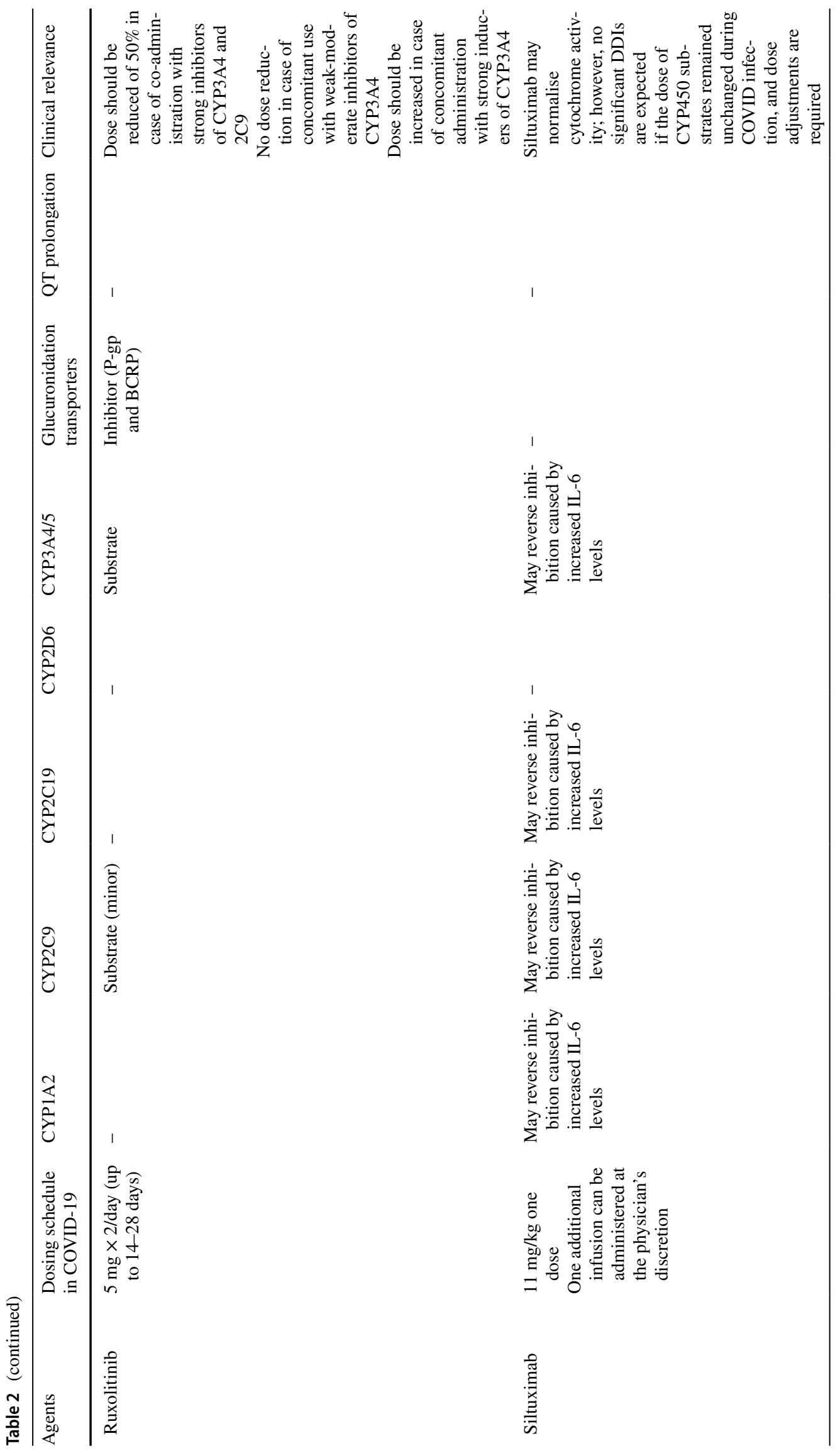

$\Delta$ Adis 


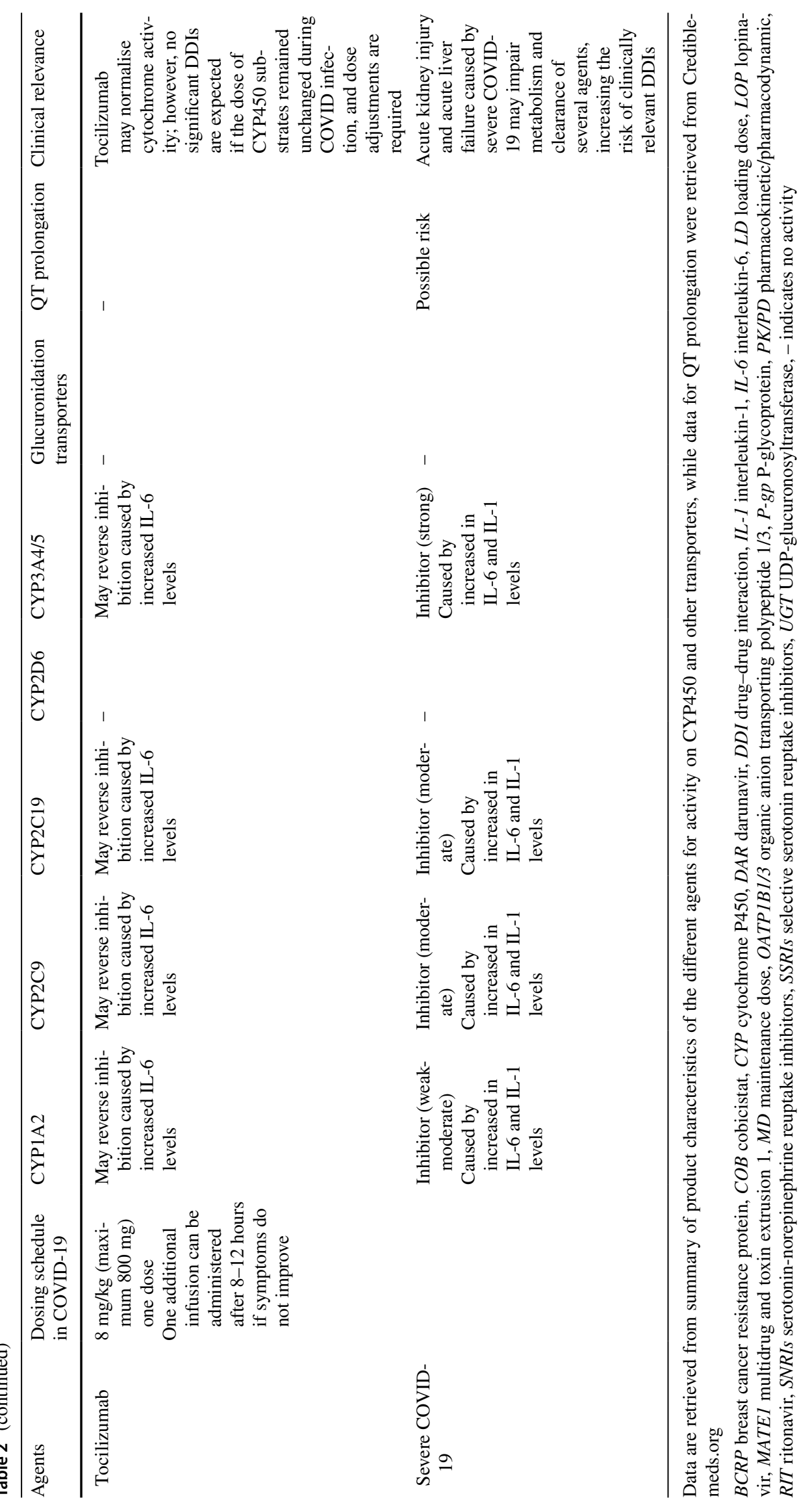




\subsection{Pharmacogenetic Issues Associated with Clinically Relevant Drug Interactions}

Finally, it should not be overlooked that the genetic polymorphisms involving CYP2C19/CYP2D6 genotypes may significantly contribute by altering the disposition of some antidepressants (e.g. escitalopram, sertraline) and antipsychotic agents (e.g. aripiprazole, haloperidol, risperidone) among CYP2C19/CYP2D6 intermediate/poor and/or ultrarapid metabolisers [40]. This may potentiate the effect of COVID-19 therapies that normally are weak or moderate inhibitors or inducers of these isozymes, thus leading to clinically relevant unpredictable interactions.

\section{Clinically Significant Interactions Between Psychotropic Agents and COVID-19 Therapies}

Several PK DDI studies between psychotropic medications and repurposed COVID-19 exist in the literature (Tables 3, $4,5,6)$. Unfortunately, most of these studies were performed in healthy volunteers before the pandemic, whereas only a minority of case reports outlined the occurrence of clinically relevant interactions between these agents among patients with COVID-19. The extent of clinical relevance based on PD effects and the need for dose adjustments reported in these studies were highly variable. Consequently, interpretation of these findings with extrapolation to patients with COVID-19 requires caution. Several limitations of these PK studies may somewhat affect the full applicability of the reported findings in the COVID-19 scenario (e.g. lack of subjects showing underlying diseases and/or polytherapy, lack of critical acute conditions). However, they may provide useful information concerning the occurrence of clinically relevant drug interactions between psychotropic medications and repurposed COVID-19 agents.

\subsection{Antidepressants}

Clinically significant drug interactions between antidepressants and COVID-19 agents, mainly antiretrovirals (ritonavir and cobicistat), were reported. Overall, 12 PK studies were performed in healthy volunteers, and two case reports and one retrospective study were also identified by our search (Table 3).

In regard to SSRIs, Gutierrez et al. [41] found no significant alteration in th edisposition of escitalopram (in terms of peak concentration $\left[C_{\max }\right]$, area under the plasma concentration-time curve [AUC] and clearance [CL]) caused by concomitant administration of ritonavir in a randomised, openlabel, three-way crossover study performed in 18 healthy volunteers. Conversely, Lorenzini et al. [42] described a case of serotonin syndrome in a patient with human immunodeficiency virus (HIV) concomitantly treated with darunavir/ ritonavir and escitalopram. Escitalopram serum concentrations were roughly three times as high as the upper threshold of the therapeutic range ( $695 \mathrm{nmol} / \mathrm{L}$ vs $250 \mathrm{nmol} / \mathrm{L}$ ), potentially due to CYP3A4 and CYP2D6 inhibition caused by ritonavir. van der Lee et al. [43] found an unexpectedly decreased paroxetine exposure (1.55-fold and 1.51-fold for AUC and $C_{\max }$, respectively) in 23 healthy volunteers concomitantly treated with fosamprenavir/ritonavir, probably related to displacement from protein binding of paroxetine resulting in higher $\mathrm{CL}$, considering that both paroxetine (95\%) and fosamprenavir/ritonavir (respectively $90 \%$ and 98\%) are highly bound to plasma proteins. Similarly, Hanan et al. [44] found an unexpected decrease in sertraline disposition (respectively 1.49-fold and 1.36-fold for AUC and $C_{\text {max }}$ ) in $31 \mathrm{HIV-infected} \mathrm{and} \mathrm{uninfected} \mathrm{subjects} \mathrm{treated}$ with ritonavir associated with different protease inhibitors (PIs), including lopinavir. The findings were attributed to induction of uridine diphosphate glucuronosyltransferase (UGT) activity. Consequently, it was concluded that higher paroxetine or sertraline dosage may be required in patients concomitantly treated with PIs and ritonavir to retain antidepressants effects. Ouellet et al. [45] found no significant alteration in ritonavir disposition among 16 healthy volunteers cotreated with the moderate CYP2D6 inhibitor fluoxetine. Consequently, it may be speculated that no lopinavir/ ritonavir dose adjustments should be required in patients with mild-to-moderate COVID-19 receiving fluoxetine treatment. Finally, in a retrospective study including 575 patients affected by venous thromboembolism and treated with fulldose enoxaparin, Samuel and Seifert [46] reported a higher risk of major bleeding in subjects treated with escitalopram compared with those receiving other SSRIs or no antidepressant therapy. This is probably due to the well-known increased risk of bleeding associated with SSRIs and resulting in additive PD toxicity [47].

Among serotonin-noradrenaline reuptake inhibitors, only one case report of interaction with COVID-19 agents was reported. Mas Serrano et al. [48] described a case of serotonin syndrome in a patient receiving lopinavir/ritonavir for COVID-19 pneumonia and concomitantly treated with duloxetine and lithium. The syndrome was attributed to the inhibitory effect of ritonavir on CYP2D6, but unfortunately a PK analysis and/or therapeutic drug monitoring (TDM) of duloxetine were not performed. However, a PD interaction also could not be ruled out.

Among TCAs, clinically significant drug interactions with repurposed agents for COVID-19 were documented in healthy volunteers only for desipramine. Mascolini et al. [49] found a moderate increase in desipramine disposition (58-65\% and $24 \%$ for AUC and $C_{\max }$, respectively) in nine healthy subjects concomitantly treated with cobicistat. 


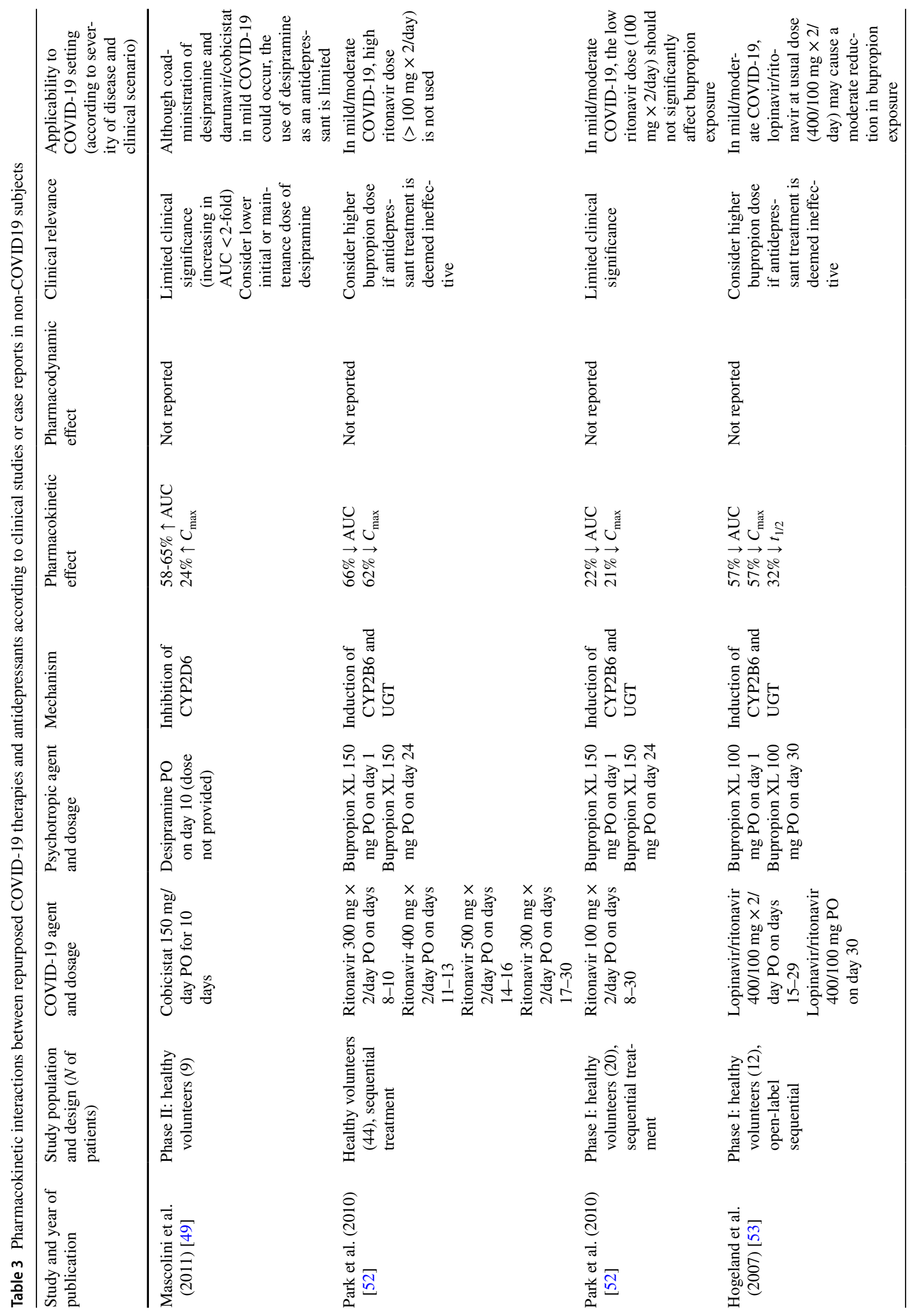




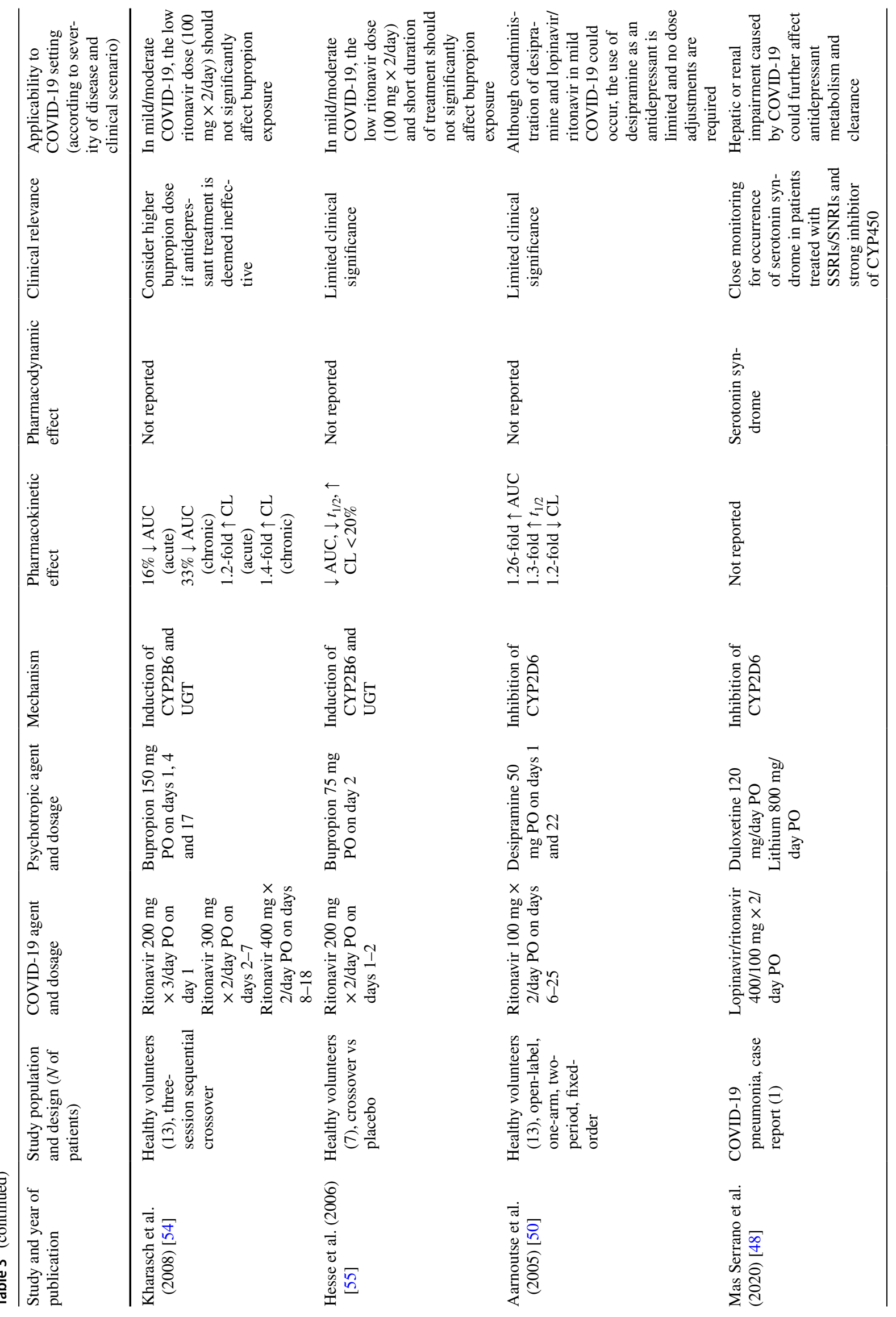




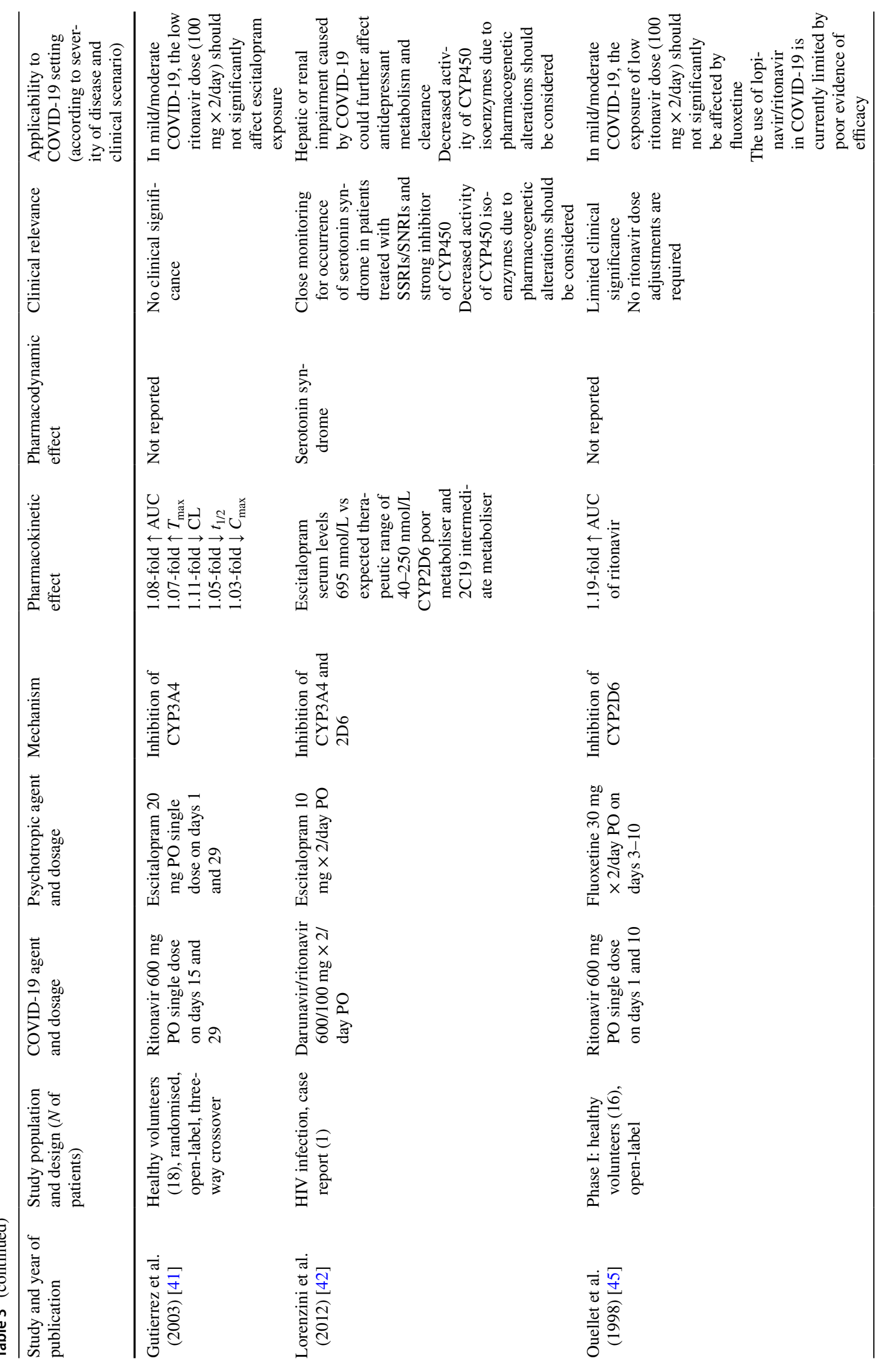




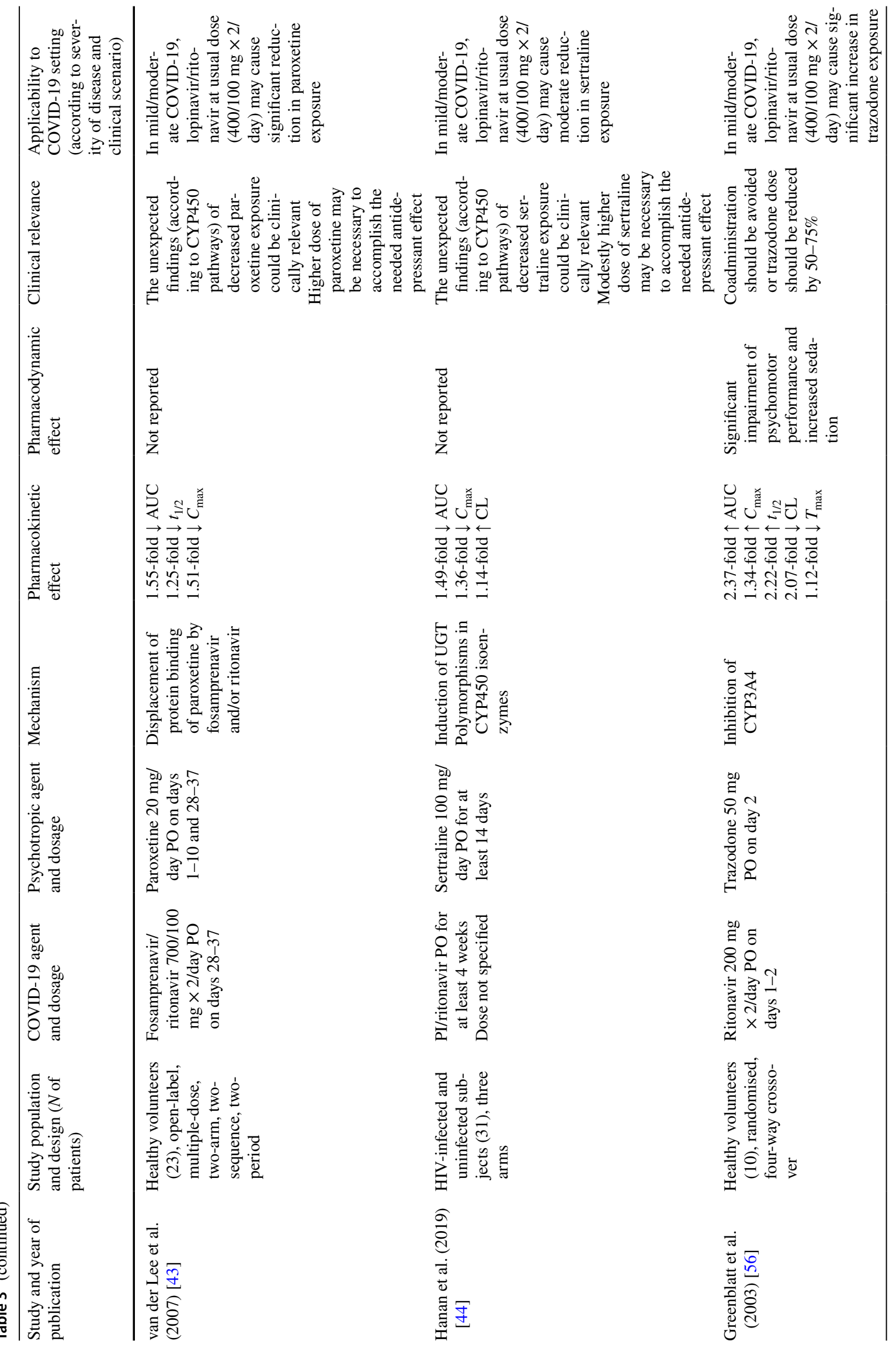




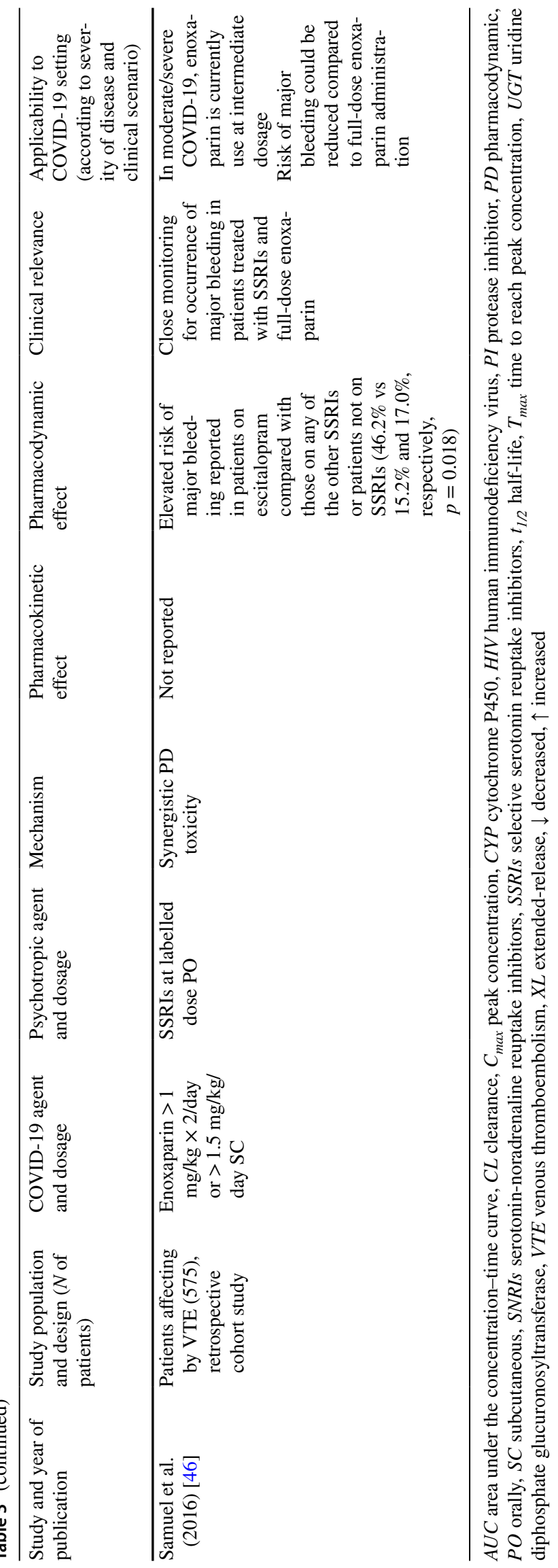




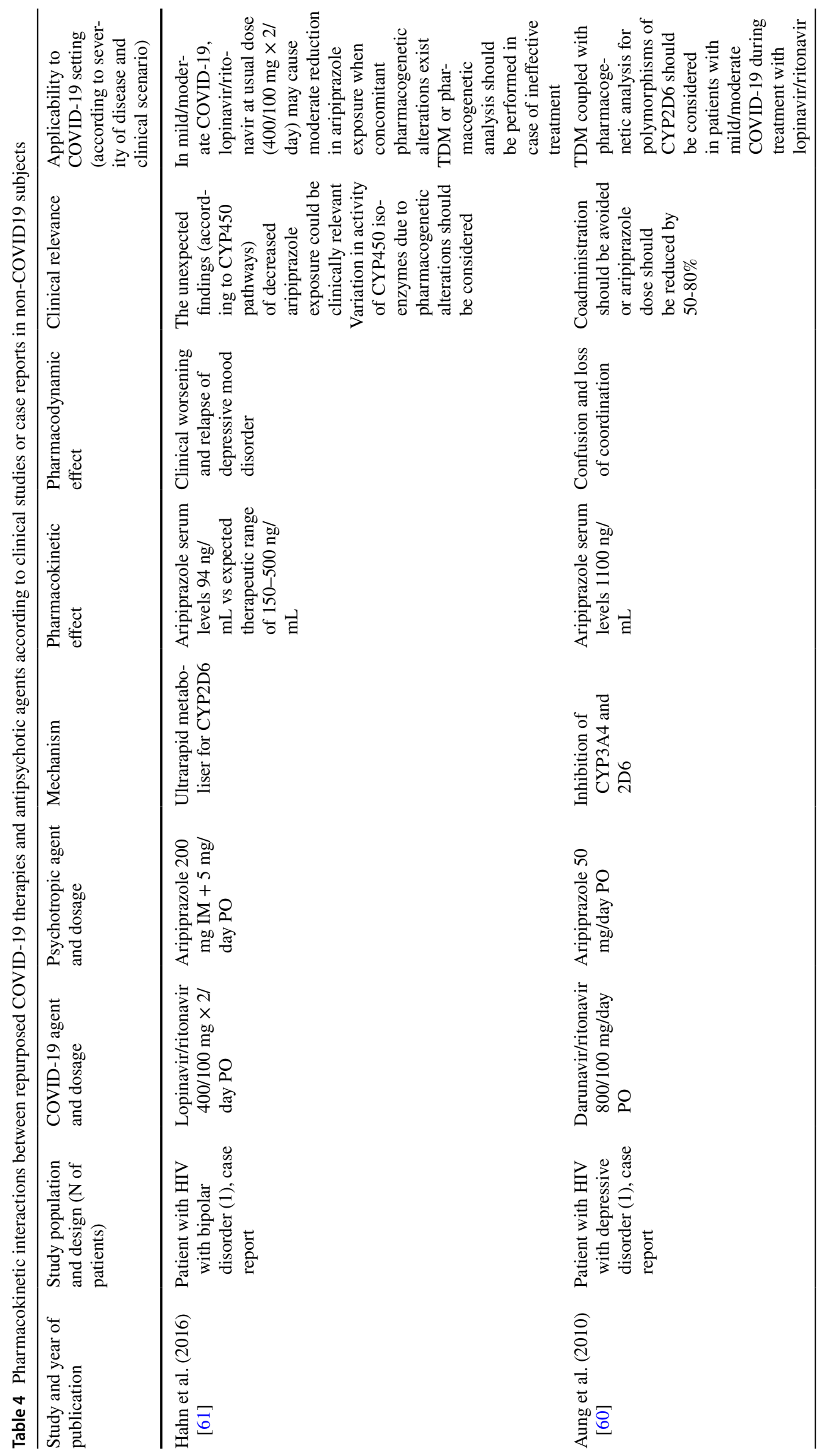




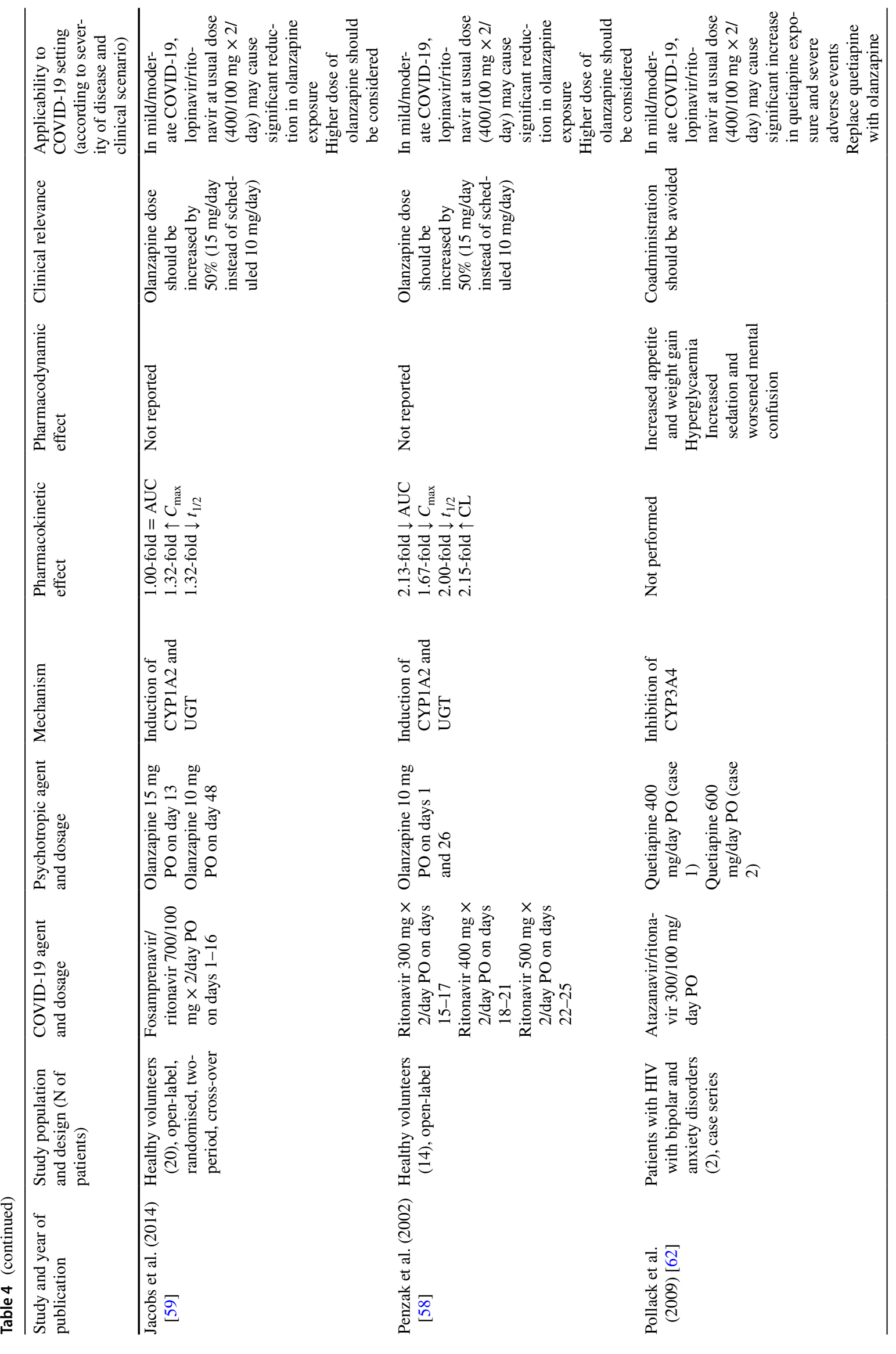




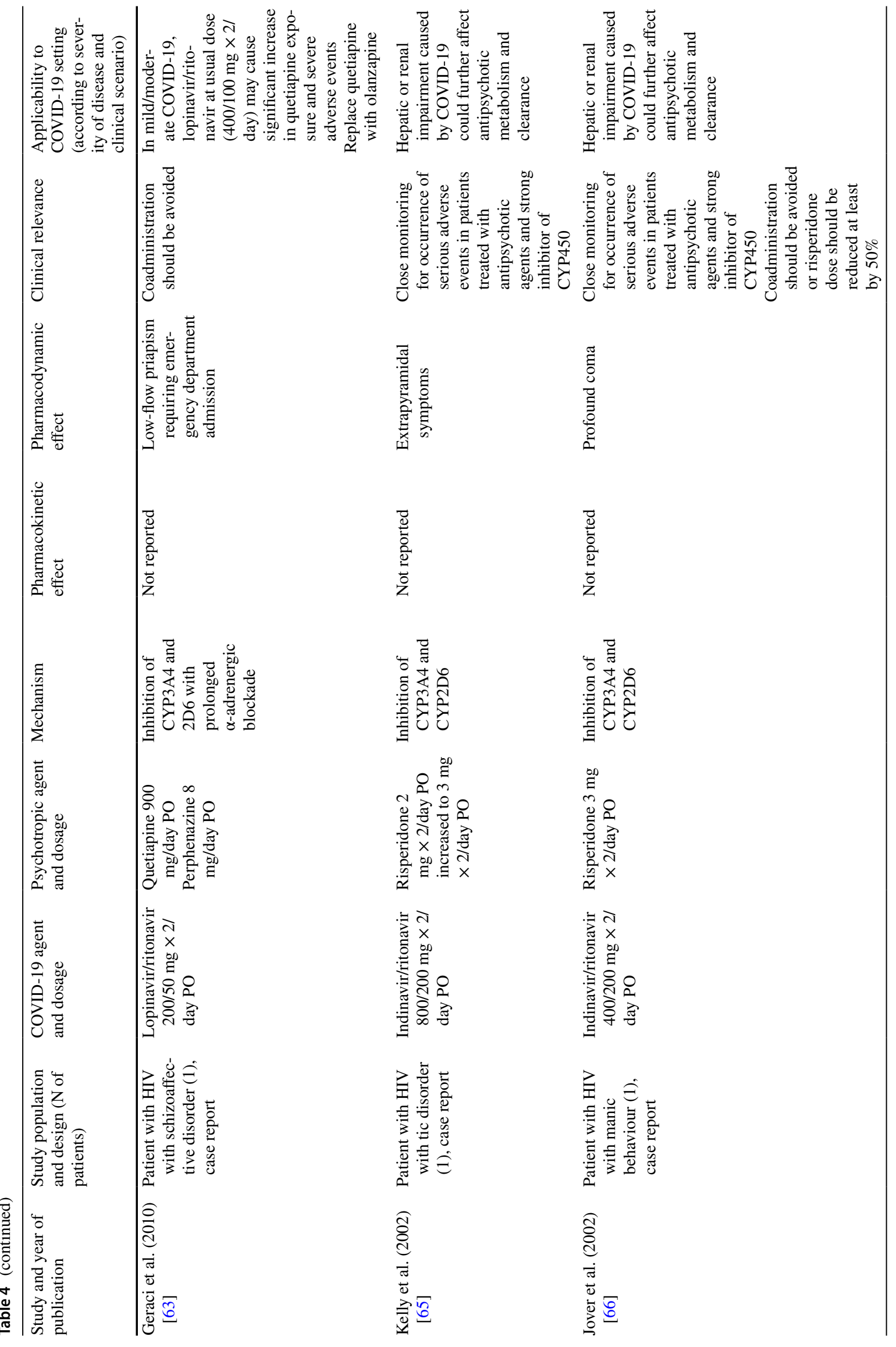




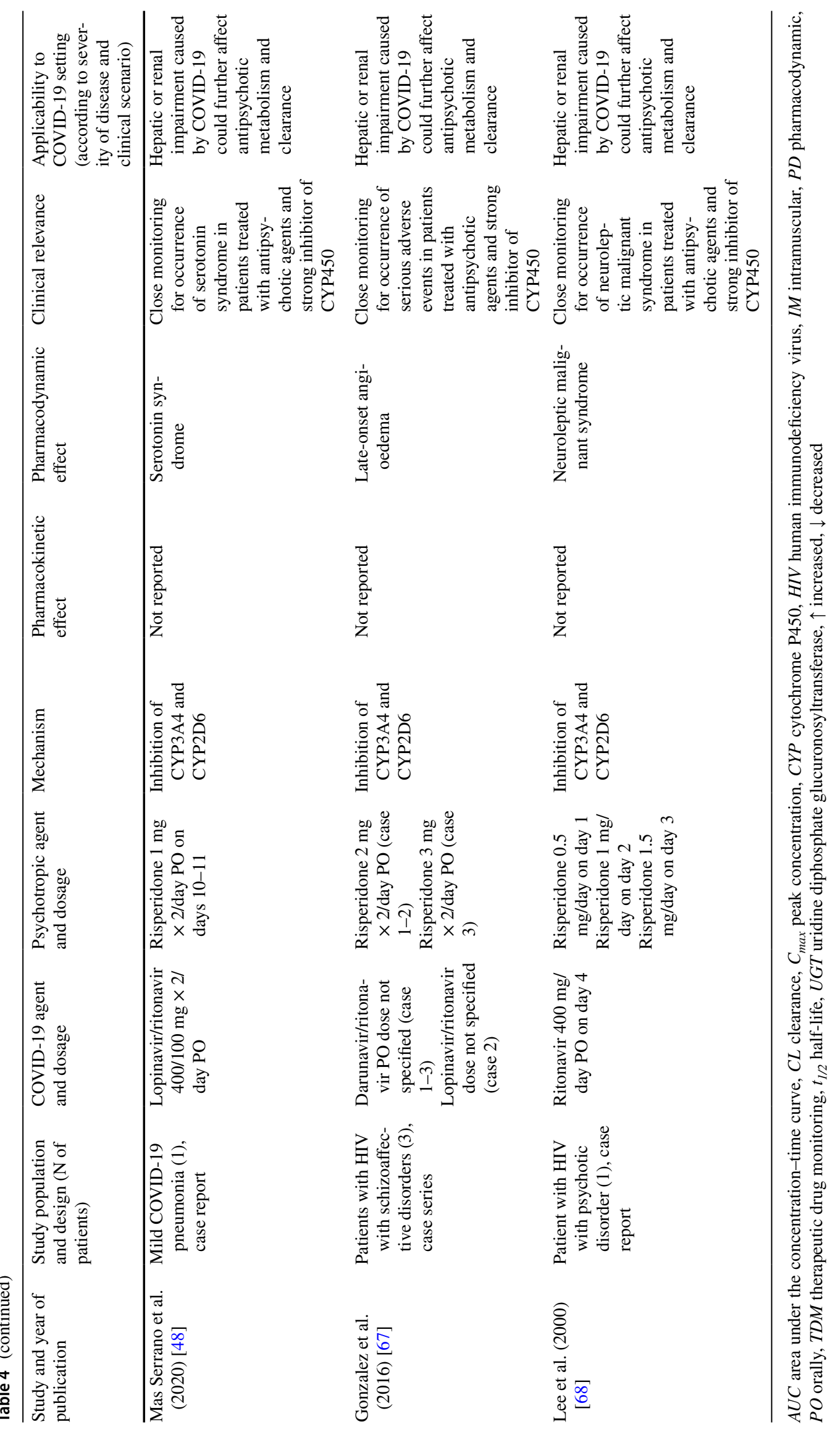




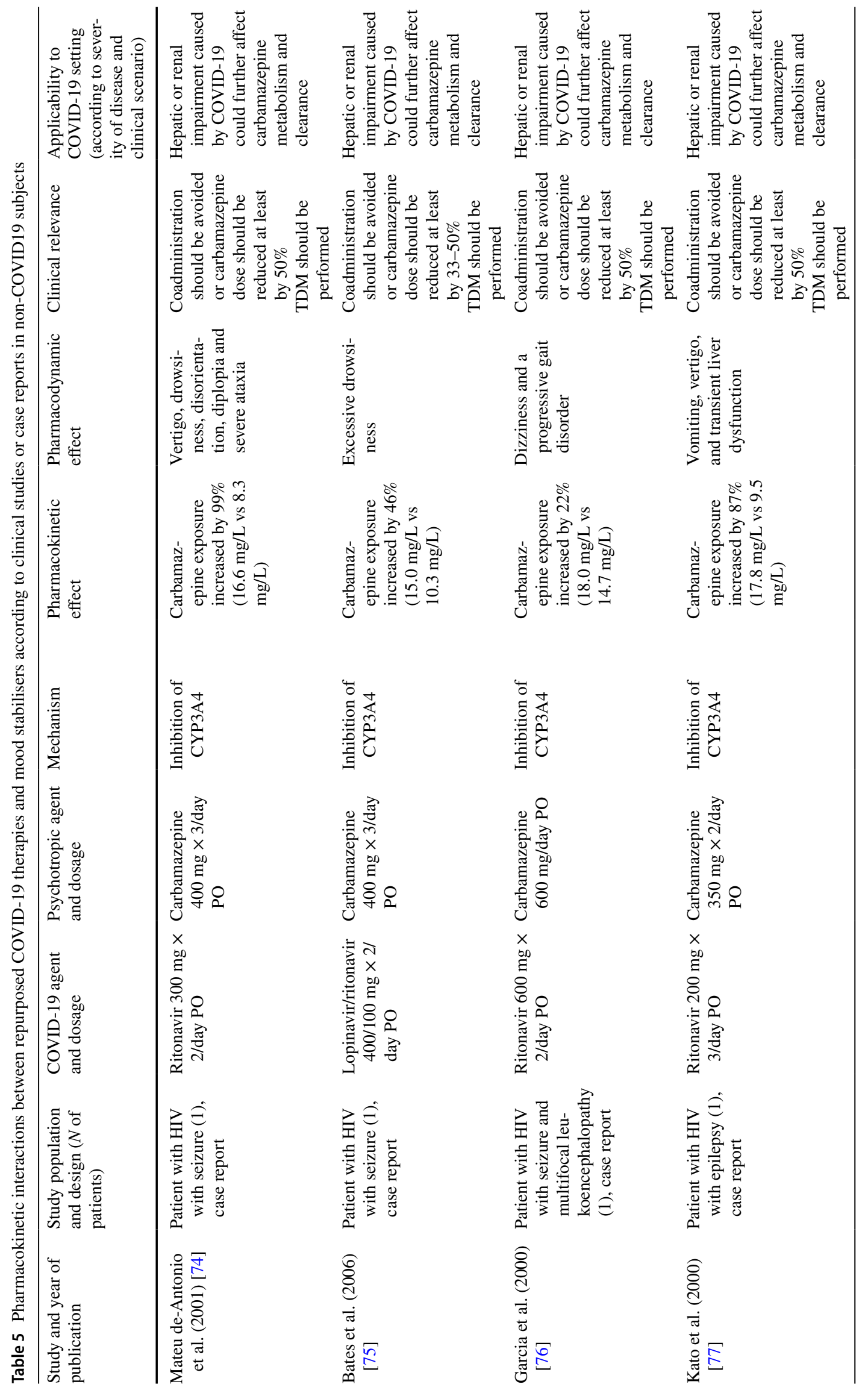




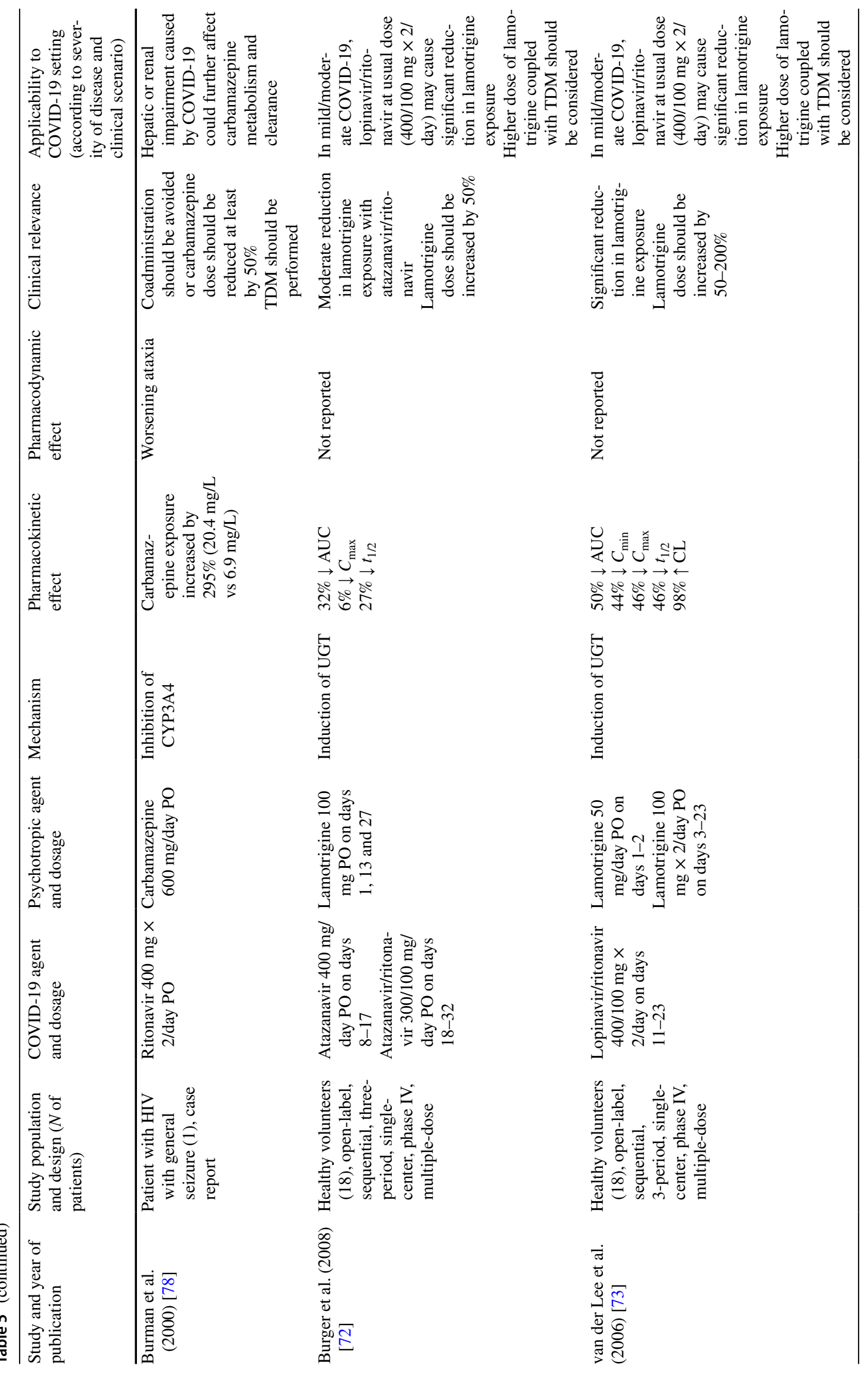




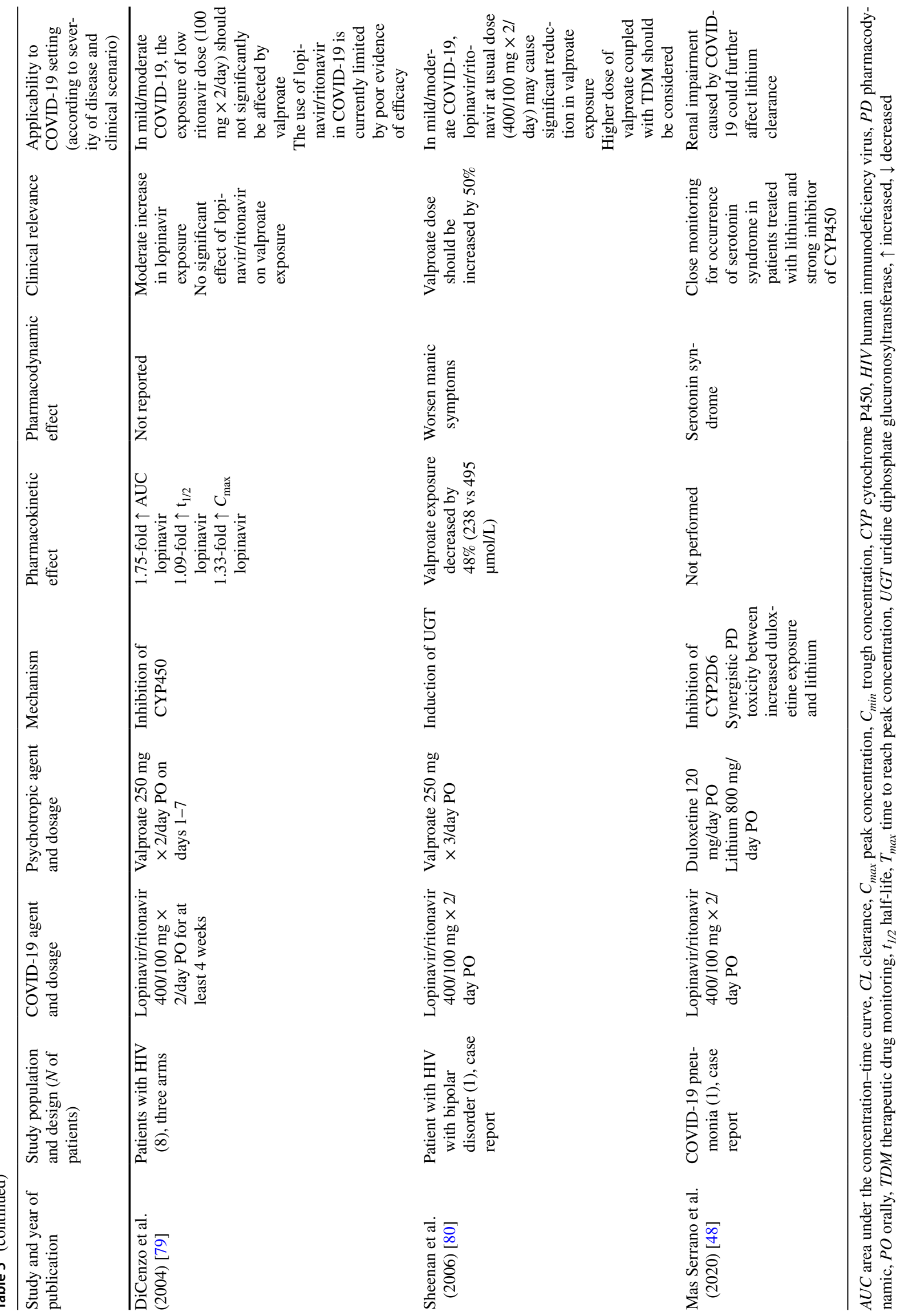




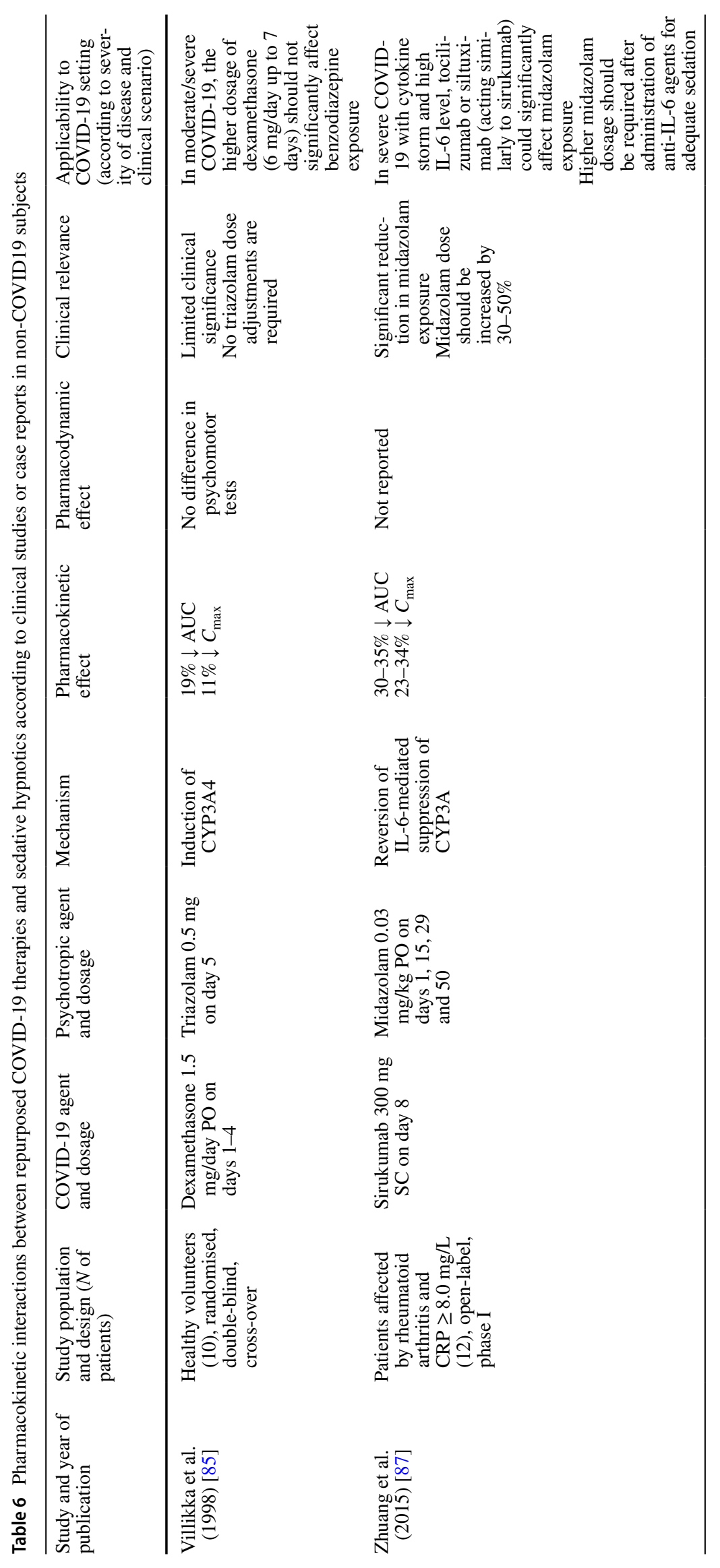




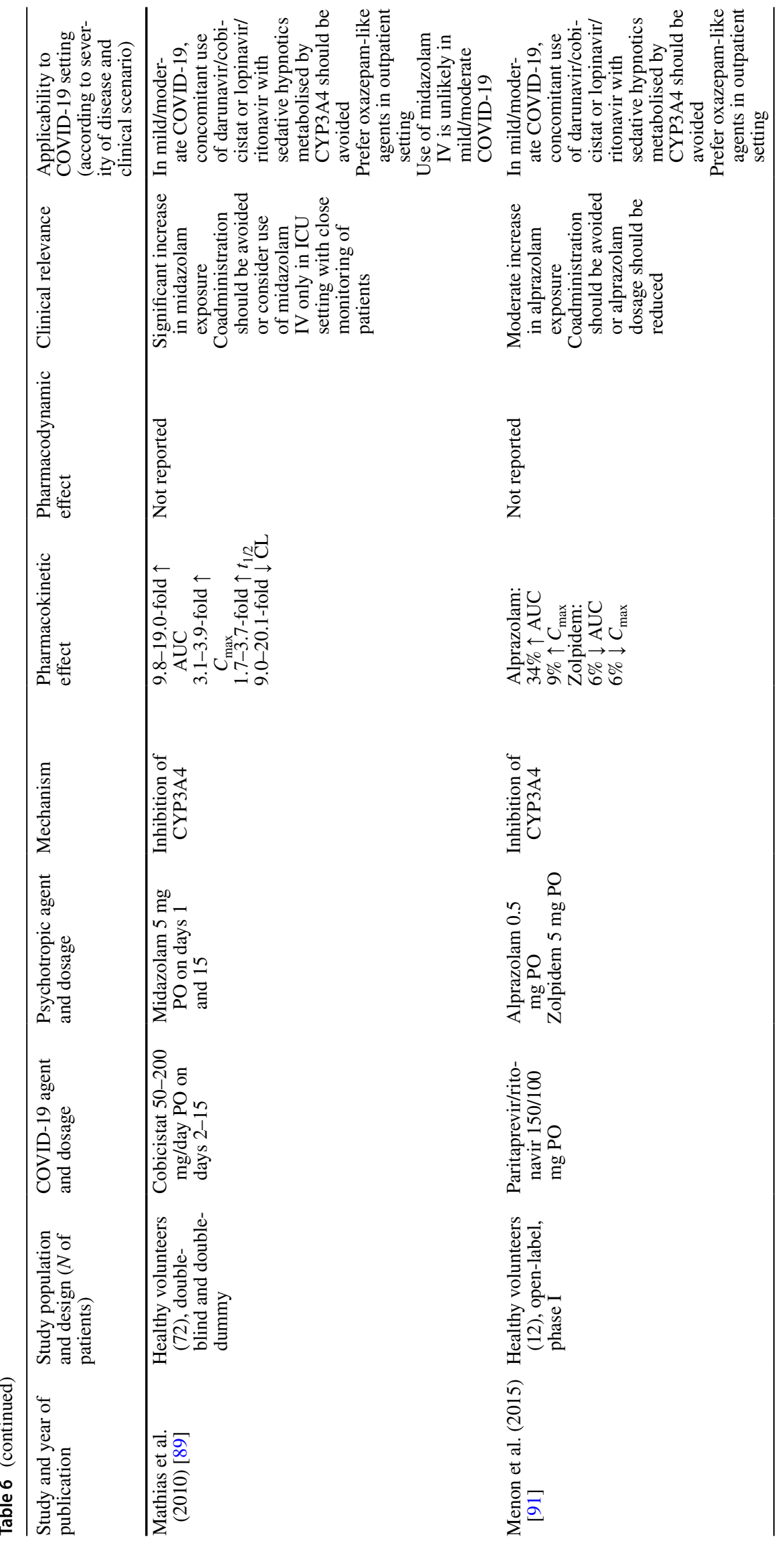




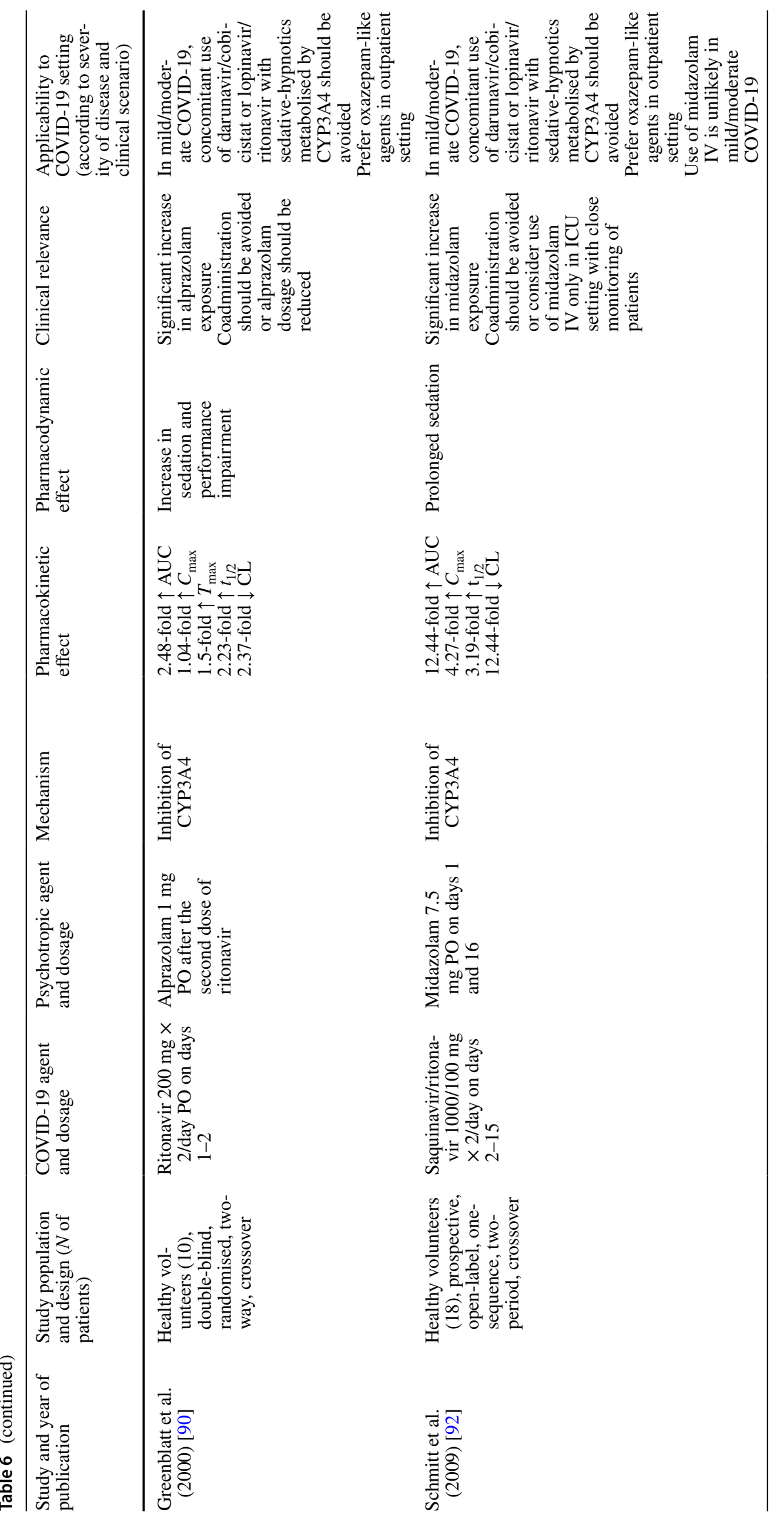




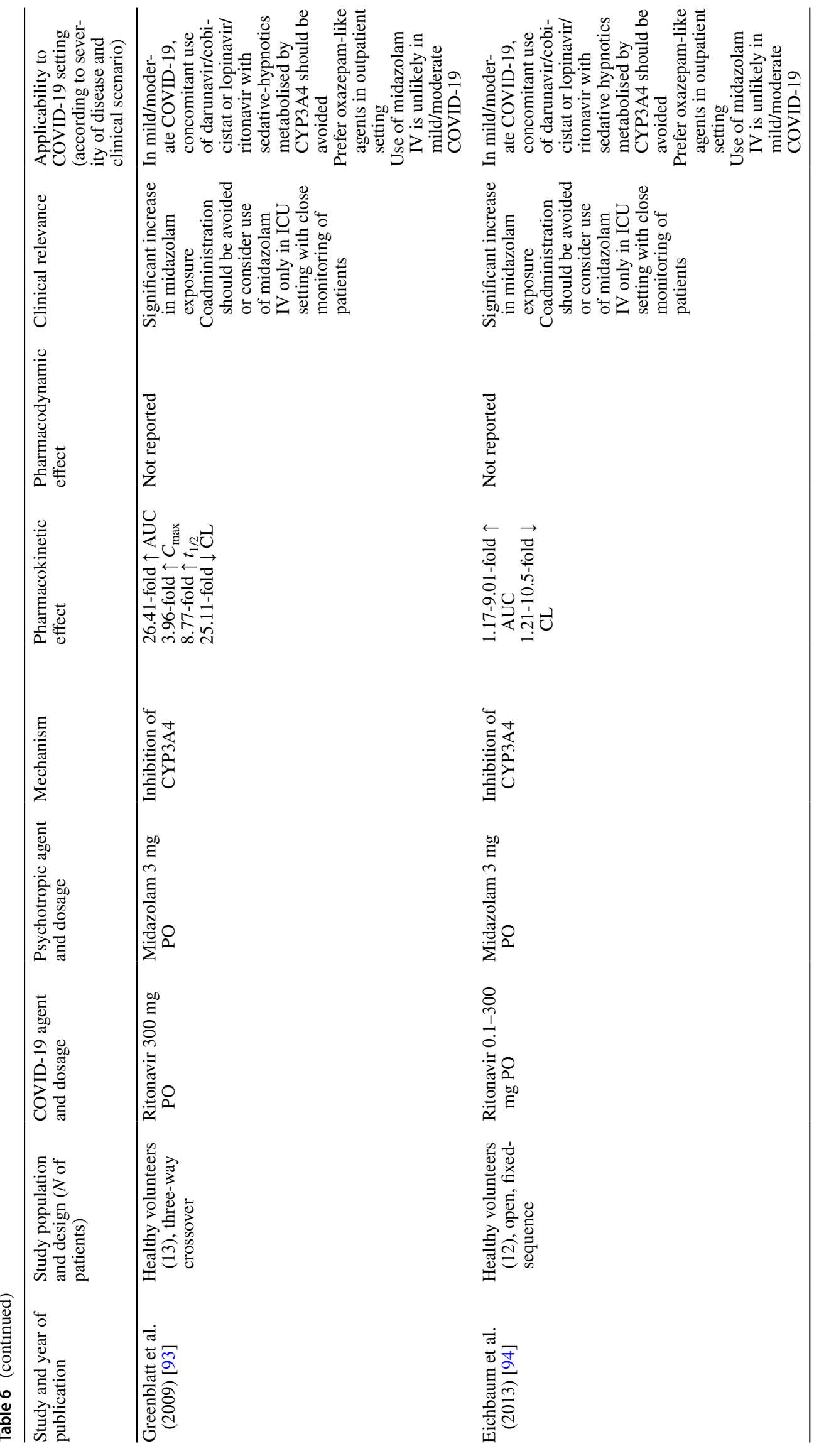




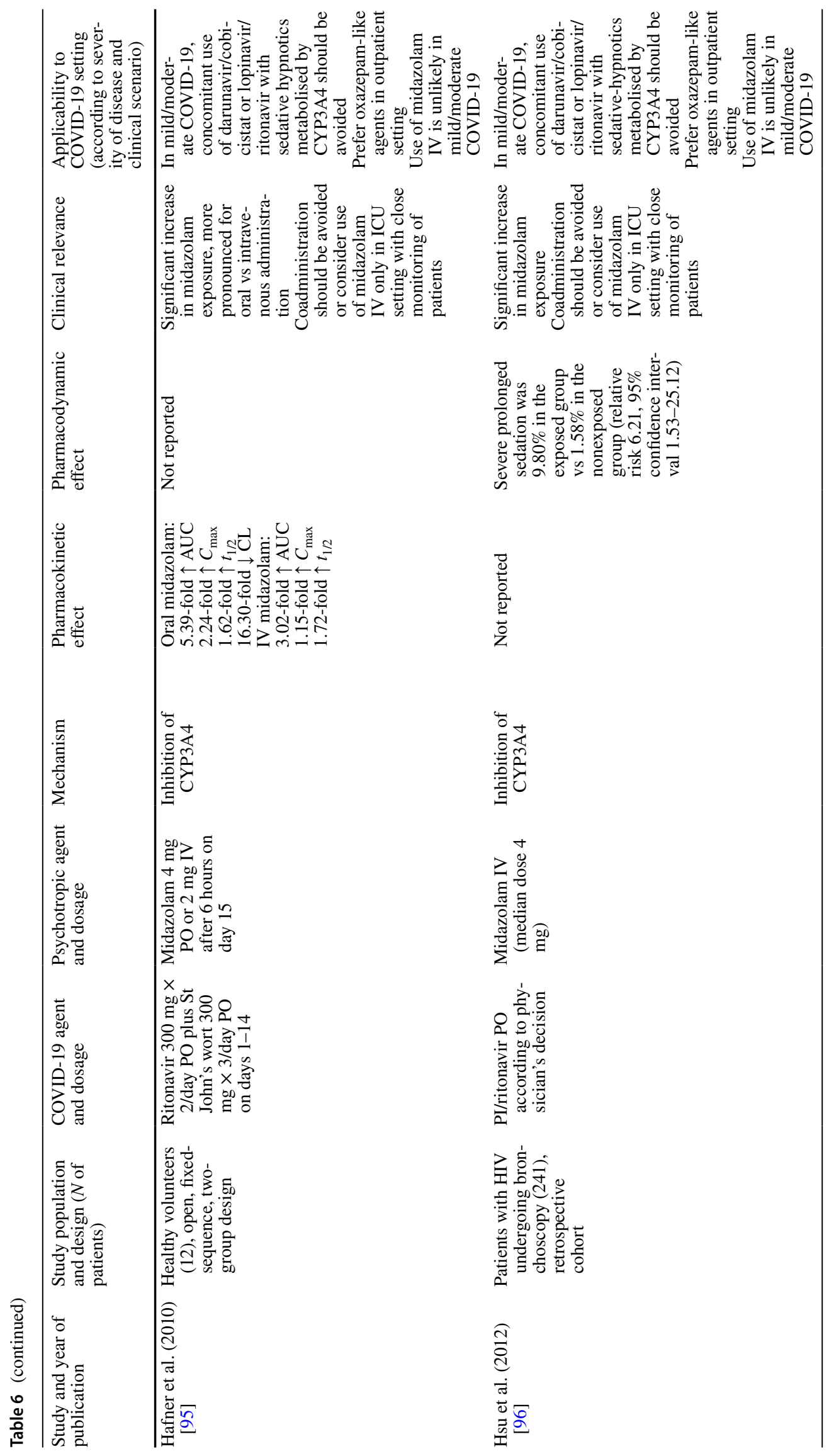




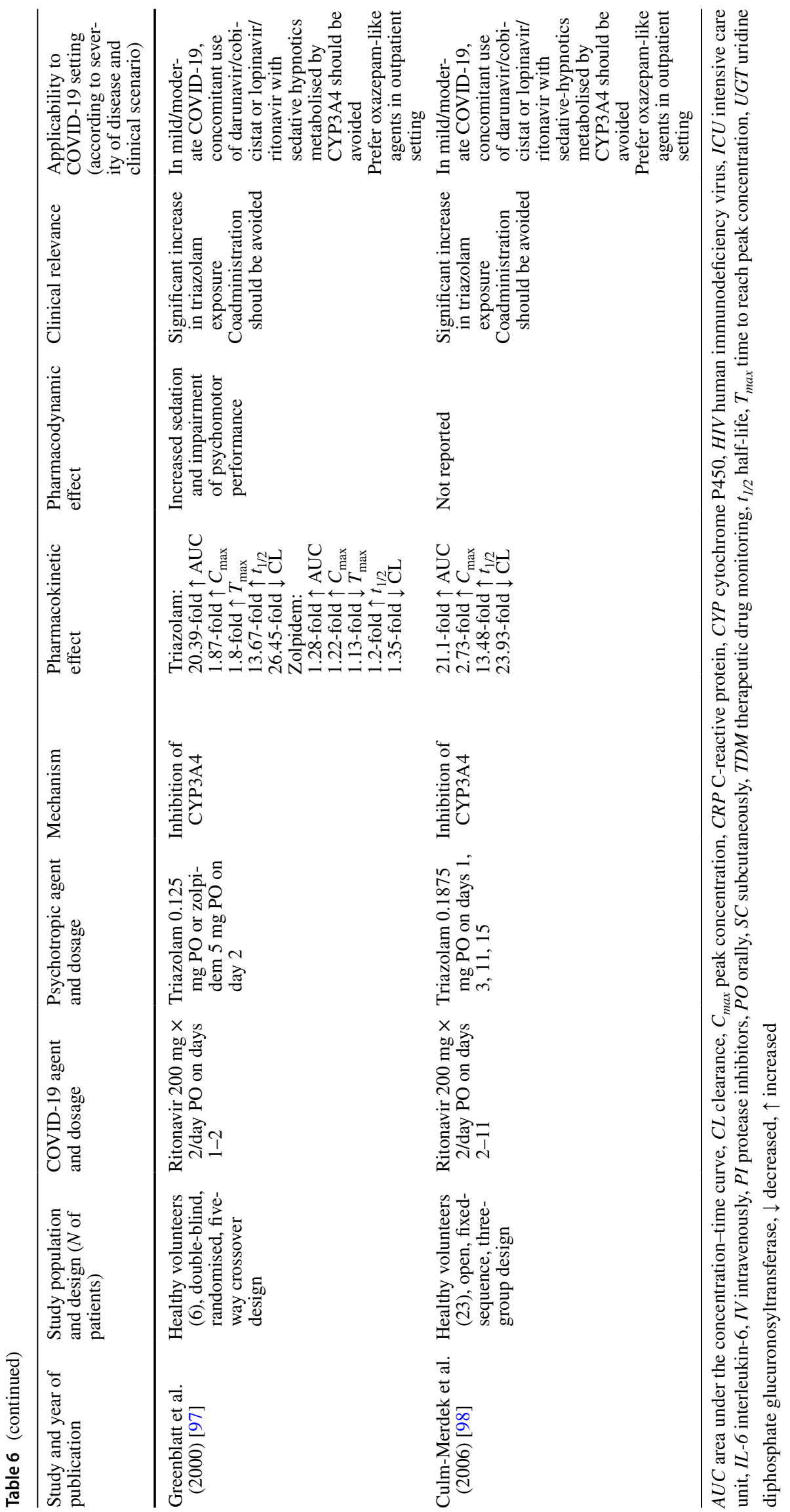


Similarly, Aarnoutse et al. [50] found a slight increase in desipramine exposure (1.26-fold in AUC) and decreased CL among 13 healthy volunteers concomitantly receiving ritonavir. The findings could be due to cobicistat- and/or ritonavir-mediated CYP2D6 inhibition and should prompt lowering of the initial or maintenance dose of desipramine in patients receiving cotreatment with these PIs. However, given the current limited use of desipramine and other TCAs as first-line treatment for depression, the relevance of these PK interactions in the COVID-19 setting is expected currently to be of limited interest [51].

Five PK studies assessed in healthy volunteers the impact of ritonavir alone and/or in combination with lopinavir on bupropion exposure [52-55]. Consistent findings were found in terms of decreased bupropion disposition, from 20 to $62-66 \%$ for $C_{\max }$ and AUC, respectively, possibly due to induction of CYP2B6 and UGT enzymes caused by ritonavir. Consequently, a higher bupropion dosage could be appropriate in patients with COVID-19 concomitantly treated with lopinavir/ritonavir whenever antidepressant treatment is deemed ineffective.

Greenblatt et al. [56] found a significant increase in trazodone exposure (respectively 2.37-fold and 2.22-fold in AUC and half-life) coupled with a 2.07-fold decreased CL among ten healthy subjects concomitantly receiving ritonavir, possibly resulting from strong inhibitory activity on CYP3A4. A significant impairment of psychomotor performance and increased sedation were also reported. Consequently, it was recommended that the coadministration of trazodone and PIs/ritonavir should be avoided or the trazodone dose should be reduced by $50-75 \%$.

No other PK DDI between antidepressants and COVID19 agents has been studied. However, based on the PK/PD properties of the different medications, it may be speculated that some clinically relevant interactions might occur. Predicted DDIs and their potential risk are summarised in Table 1 of the Electronic Supplementary Material (ESM). No further high-risk PK or PD interactions between antidepressants and COVID-19 therapies can be predicted. For lopinavir/ritonavir and for darunavir/cobicistat, a moderate risk of relevant PK interactions (higher exposure of antidepressants metabolised by CYP3A4 and CYP2D6 requiring dose adjustments) and PD (QT prolongation with concomitant administration of lopinavir/ritonavir and several SSRIs, TCAs, mianserin, mirtazapine, or trazodone) interactions could be predicted. In patients affected by a cytokine storm and treated with anti-IL-6 and/or anti-IL-1 agents such as tocilizumab, siltuximab and canakinumab, an upregulation of CYP3A4 activity may be expected [57]. Consequently, for antidepressants that are metabolised by CYP3A4, it might be necessary to increase the dosage for ensuring efficacy, even if the magnitude of this increase is difficult to predict a priori. However, it should be mentioned that anti-IL-6 and IL-1 agents are used in patients with moderate-to-severe COVID19 admitted to the ICU in which oral antidepressants are usually stopped. No clinically relevant interactions involving remdesivir, colchicine, ruxolitinib, dexamethasone, bamlanivimab and casirivimab/imdevimab are expected to occur with antidepressants.

Overall, according to real-world evidence and PK/PD properties, lopinavir/ritonavir and darunavir/cobicistat pose the major concerns for the occurrence of clinically relevant interactions with antidepressants. Higher exposure may occur for TCAs, serotonin-noradrenaline reuptake inhibitors (except duloxetine), SSRIs (except sertraline and vortioxetine) and other antidepressants (except milnacipran). A significant decrease in bupropion exposure was reported. However, no antidepressant should be a priori avoided in the case of concomitant administration with PIs. The use of agents showing a low risk of PK/PD interactions (e.g. sertraline, vortioxetine, milnacipran) should be preferred.

\subsection{Antipsychotic Agents}

Clinically significant drug interactions between antipsychotic agents and COVID-19 agents, especially with ritonavir-boosted PIs, were documented in healthy volunteers and in patients without COVID-19. Overall, two PK studies and nine case reports were identified by our search (Table 4). None of these involved first-generation antipsychotic agents.

Penzak et al. [58] found a significant decrease in olanzapine exposure (respectively 2.13 -fold and 1.67 -fold in AUC and $\left.C_{\max }\right)$ coupled with increased CL (2.15-fold) in 14 healthy volunteers concomitantly treated with ritonavir, probably due to CYP1A2 and UGT induction. Consequently, a $50 \%$ dosage increase of olanzapine $(15 \mathrm{mg} /$ day instead of scheduled $10 \mathrm{mg} /$ day) was suggested in patients cotreated with ritonavir-boosted PIs. These findings were also confirmed by Jacobs et al. [59] in a PK study performed in 20 healthy subjects concomitantly treated with fosamprenavir/ritonavir and olanzapine. No significant difference in olanzapine exposure was found when concomitantly administered with fosamprenavir/ritonavir (at a dosage of $15 \mathrm{mg}$ /day) or used alone (at a dosage of $10 \mathrm{mg} /$ day), thus proving the need for increasing olanzapine dose.

Aung et al. [60] described a case of aripiprazole toxicity (resulting in confusion and loss of motor coordination) in a patient with HIV treated with darunavir/ritonavir and affected by depressive disorder. The aripiprazole serum level was more than two times higher than the upper threshold of the desired range $(1100 \mathrm{ng} / \mathrm{mL}$ vs $500 \mathrm{ng} /$ $\mathrm{mL}$ ). This was attributed to the inhibitory activity of ritonavir on CYP3A4- and CYP2D6-mediated metabolism of aripiprazole and called for an aripiprazole TDM-based dose adjustment (reduction by $50-80 \%$ ), or avoidance of coadministration. Interestingly, opposite findings were 
reported by Hahn and Roll [61] in a patient with HIV affected by bipolar disorder who experienced a relapse of mood disorder associated with an aripiprazole (both oral and intramuscular depot formulations) serum concentration below the therapeutic range ( $94 \mathrm{ng} / \mathrm{mL}$ vs $150-500$ $\mathrm{ng} / \mathrm{mL}$ ) during cotreatment with lopinavir/ritonavir. The findings were attributed to the patients' status of extensive or ultrarapid metaboliser of CYP2D6 that was able to overcome the inhibition of CY2D6 and 3A4 activity promoted by ritonavir. It was concluded that TDM coupled with a pharmacogenetic analysis may be helpful in tailoring the aripiprazole dosage in a patient cotreated with ritonavirboosted PIs.

Pollack et al. [62] described two cases of quetiapine toxicity (increased sedation, worsened mental confusion, hyperglycaemia, weight gain) among patients with HIV with bipolar disorder concomitantly treated with atazanavir/ ritonavir. This was potentially due to the inhibitory activity on CYP3A4, although a confirmatory PK analysis and/ or quetiapine TDM were not performed. Similarly, Geraci et al. [63] reported a case of priapism in a patient with HIV affected by schizoaffective disorder treated with quetiapine and perphenazine concomitantly receiving lopinavir/ritonavir. The inhibitory activity of ritonavir on CYP3A4 and 2D6 may have increased antipsychotic agent concentrations potentially leading to prolonged $\alpha$-adrenergic blockade and consequent low-flow priapism. According to the summary of product characteristics, quetiapine dose should be reduced by one-sixth in the case of co-administration with strong CYP3A4 inhibitors such as ritonavir, to prevent the occurrence of life-threatening adverse events (e.g. QT prolongation). Olanzapine should be considered as an alternative agent to replace quetiapine according to PK/PD properties in patients affected by COVID-19 treated with lopinavir/ ritonavir [64].

Several case reports described the occurrence of lifethreatening events associated with risperidone toxicity (serotonin syndrome, neuroleptic malignant syndrome, profound coma, extrapyramidal symptoms and late-onset angioedema) in patients with HIV treated with different ritonavir-boosted PIs [48, 65-68]. The underlying mechanism is probably due to inhibitory activity of ritonavir on CYP3A4 and CYP2D6, although in no cases was a PK analysis or TDM performed to prove risperidone overexposure. Notably, risperidone toxicity was also reported in a case of mild COVID-19 pneumonia requiring 14 days of lopinavir/ritonavir-based therapy [48].

Although the potential of DDIs of other antipsychotic agents with COVID-19 therapies has not been investigated in humans, clinically relevant interactions may be predicted according to the PK/PD properties of the different agents (Table 2 of the ESM). High-risk PK or PD interactions between antipsychotic agents (namely haloperidol, pimozide, sertindole, and ziprasidone) and antiretrovirals (darunavir/cobicistat or lopinavir/ritonavir) have been detected, mainly associated with an increase in antipsychotic exposure leading to life-threatening events (e.g. neuroleptic malignant syndrome, fatal arrhythmias caused by QT prolongation) $[69,70]$. Consequently, coadministration should be cautiously avoided or antipsychotic dosage should be reduced (e.g. by one-sixth for quetiapine or $50 \%$ for iloperidone) [71]. Interestingly, a decrease in olanzapine exposure may occur during cotreatment with lopinavir/ritonavir, due to induction of CYP1A2 and UGT enzymes, possibly requiring an olanzapine dose increase [64]. The risk of PK or PD interactions between lopinavir/ritonavir and other antipsychotic agents may be moderate, and mainly concerns the risk of QT prolongation exhibited by each specific agent. Similarly, darunavir/cobicistat may increase the exposure of several antipsychotic agents metabolised by CYP3A4, thus requiring dose adjustments $[69,70]$. According to CYP status, paliperidone and ziprasidone may be optimal alternatives to minimise the occurrence of clinically relevant interactions with PIs, although ziprasidone poses a high risk of QT prolongation in patients concomitantly treated with lopinavir/ritonavir.

In patients affected by a cytokine storm and treated with anti-IL-6 and/or anti-IL-1 agents such as tocilizumab, siltuximab and canakinumab, an upregulation of CYP3A4 activity may be expected [57]. Consequently, for those antipsychotic agents that are metabolised by CYP3A4, it might be necessary to increase the dosage to ensure efficacy, even if the magnitude of this increase is difficult to predict a priori. However, it should be mentioned that anti-IL- 6 and IL-1 agents are used in patients with moderate-to-severe COVID19 admitted to the ICU in which oral antipsychotic agents are usually stopped. Conversely, haloperidol is the only antipsychotic agent for which the magnitude of the effect could be clinically relevant in the ICU setting. When administered parenterally, a dosage increase could be needed to ensure adequate anti-delirium efficacy under these circumstances, closely monitoring QT prolongation and occurrence of torsade de pointes, regardless of potential drug interactions. Indeed, some patients may receive long-acting injectable antipsychotic agents to manage schizophrenia or bipolar disorder. In this scenario, clinically relevant drug interactions may also occur despite ICU admission because of the long elimination half-life of these agents.

Potential additive haematological toxicity should be considered when clozapine is used with tocilizumab, siltuximab, canakinumab and ruxolitinib. Additionally, higher clozapine concentrations could be detected in severe COVID-19 forms due to downregulation of CYP1A2 caused by pro-inflammatory cytokines, leading to potential life-threatening toxicity [21]. Notably, given that thioridazine is a moderate inducer of CYP3A4, a doubling of the daily dose of COVID-19 
agents metabolised through this pathway should be consider (colchicine $1 \mathrm{mg} \times 2$, ruxolitinib $10 \mathrm{mg} \times 2$, or dexamethasone $12 \mathrm{mg}$ instead of $6 \mathrm{mg}$ ).

No clinically relevant interactions involving remdesivir, enoxaparin, bamlanivimab and casirivimab/imdevimab are expected to occur with antipsychotic agents according to $\mathrm{PK} / \mathrm{PD}$ properties. Overall, according to real-world evidence and PK/PD properties, lopinavir/ritonavir and darunavir/ cobicistat pose the major concerns for the occurrence of clinically relevant interactions with antipsychotic agents. Higher exposure may occur for most of the first-generation and second-generation antipsychotic agents. Concomitant administration of PIs and haloperidol, quetiapine and pimozide should be avoided considering the significant increase in exposure. Ziprasidone and sertindole should be avoided according to the high risk of QT prolongation when concomitantly used with lopinavir/ritonavir. Haloperidol could be used in the ICU setting under strict monitoring. Olanzapine should be preferred as first-line therapy for the management of agitated delirium. Paliperidone could be used as a firstline alternative in severe psychosis according to its low risk of $\mathrm{PK} / \mathrm{PD}$ interactions.

\subsection{Mood Stabilisers}

Clinically significant drug interactions between mood stabilisers and repurposed COVID-19 agents, especially with ritonavir-boosted PIs, were documented in healthy volunteers and in patients without COVID-19. Overall, two PK studies and eight case reports were identified by our search (Table 5).

Burger et al. [72] found a moderate reduction in lamotrigine exposure (32\% in AUC) in 18 healthy volunteers concomitantly treated with atazanavir/ritonavir, due to an UGT induction. Similarly, van der Lee et al. [73] reported a significant decrease in lamotrigine disposition $(50 \%$ in AUC and $46 \%$ in $C_{\max }$ ) coupled with a $98 \%$ increase in drug CL among 18 healthy subjects concomitantly receiving lopinavir/ritonavir. Overall, the findings support the need for a lamotrigine dosage increase of $50-200 \%$ in patients cotreated with ritonavir-boosted PIs, and suggest that TDM may be a helpful tool in achieving adequate lamotrigine serum concentrations.

Several case reports described the occurrence of carbamazepine toxicity (e.g. vertigo, ataxia, drowsiness) in patients with HIV affected by epilepsy or seizure receiving ritonavir alone or in combination with lopinavir [74-78]. Therapeutic drug monitoring demonstrated a significant increase in carbamazepine serum concentrations, from 22 to $295 \%$, due to inhibitory activity of ritonavir on CYP3A4. Coadministration of carbamazepine and ritonavir should be avoided or the carbamazepine dose should be reduced by at least 50\% using a TDM-guided approach.
DiCenzo et al. [79] found a moderate increase in lopinavir exposure (respectively 1.75-fold and 1.33-fold in AUC and $C_{\max }$ ) in eight patients with HIV concomitantly receiving valproate, while no significant variations in valproate disposition were caused by lopinavir/ritonavir. Conversely, Sheenan et al. [80] reported a significant decrease in valproate disposition in a patient with HIV affected by bipolar disorder concomitantly treated with lopinavir/ritonavir, responsible for the worsening of manic symptoms. Valproate serum concentrations were decreased by $48 \%$ (238 vs 495 $\mu \mathrm{mol} / \mathrm{L}$ ), possibly owing to the induction of UGT caused by lopinavir/ritonavir. Increase in valproate dosage may be needed in patients treated with ritonavir and PIs, and TDM is strongly suggested to assess the achievement of adequate valproate serum concentrations [80,81]. A case report of serotonin syndrome in a patient affected by COVID-19 pneumonia treated with lopinavir/ritonavir and concomitantly receiving lithium and duloxetine was described [48], although unfortunately no PK analysis and/or TDM of lithium was performed.

A summary of predicted PK and PD interactions between mood stabilisers and other COVID-19 agents is shown in Table 3 of the ESM. Apart from the increased risk of thrombocytopenia in patients concomitantly treated with valproate and enoxaparin due to additive toxicity [82], and a potential electrolyte imbalance caused by dexamethasone that could impair lithium renal excretion (although the clinical relevance is questionable) [83], carbamazepine poses major issues when concomitantly used with several COVID-19 agents. The coadministration of carbamazepine with lopinavir/ritonavir or with darunavir/cobicistat should be avoided if not strictly necessary, or close monitoring of carbamazepine serum concentrations coupled with appropriate dose adjustments should be implemented. Given the strong inducer activity of carbamazepine on CYP3A4 [84], a significant reduction in the exposure of several substrates of this isozyme is expected, including remdesivir, colchicine, ruxolitinib and dexamethasone. An increased dosage of these agents should be considered.

In patients affected by a cytokine storm and treated with anti-IL-6 and/or anti-IL1 agents such as tocilizumab, siltuximab and canakinumab, an upregulation of CYP3A4 activity may be expected [57]. Consequently, as carbamazepine is metabolised by both CYP3A4 and CYP2C $9 / 19$, it might be necessary to increase its dosage to ensure efficacy, even if the magnitude of this increase is difficult to predict a priori. No clinically relevant interactions between other mood stabilisers and COVID-19 therapies (excluded lopinavir/ritonavir) are expected according to PK/PD properties of the different agents.

Overall, according to real-world evidence and PK/ PD properties, carbamazepine poses the major concern for the occurrence of clinically relevant interactions with 
COVID-19 agents, owing to strong induction of CYP3A4 activity. Furthermore, higher carbamazepine concentrations may occur in patients receiving lopinavir/ritonavir and/or darunavir/cobicistat, and coadministration should be avoided. If carbamazepine is strictly required (e.g. severe bipolar disorder in long-term therapy), dose adjustment (reduction by one-third) coupled with TDM should be performed. A double dose of lamotrigine or valproic acid is required in patients co-treated with lopinavir/ritonavir owing to induction of UGT activity.

\subsection{Sedative Hypnotics}

Clinically significant drug interactions between sedative hypnotics and COVID-19 agents, especially with ritonavirboosted PIs, were documented in healthy volunteers and in patients without COVID-19. Overall, 11 PK studies mainly in healthy volunteers and one retrospective study were identified by our search (Table 6).

Villikka et al. [85] found a limited decrease in triazolam exposure (respectively $19 \%$ and $11 \%$ in AUC and $C_{\max }$ ) in ten healthy volunteers concomitantly treated with dexamethasone. Furthermore, no differences in psychomotor tests were reported. No dose adjustments should be required for triazolam or other sedative hypnotics in patients cotreated with dexamethasone, given that its moderate inducer activity on CYP3A4 occurs at high doses (above 16-24 mg/day) [86].

Zhuang et al. [87] showed a significant decrease in midazolam exposure (respectively, $34 \%$ and $35 \%$ in $C_{\max }$ and AUC) after oral administration among 12 patients with arthritis with C-reactive protein levels above $8.0 \mathrm{mg} / \mathrm{L}$ who were cotreated with the anti-IL-6 agent sirukumab. The findings were due to a reversal of the inhibitory activity promoted by this proinflammatory cytokine on CYP3A4 isozyme. Consequently, an increase in midazolam dose could be necessary in patients with severe COVID-19 and an associated cytokine storm when concomitant treatment with anti-IL-6 (i.e. tocilizumab, siltuximab), to achieve adequate sedation. The magnitude of this increase is difficult to predict a priori according to the different midazolam formulations (oral vs parenteral) and degree of disease severity (IL-6 levels) [88].

Mathias et al. [89] found a significant increase in midazolam exposure (respectively, up to 3.9-fold and 19-fold for $C_{\max }$ and AUC) coupled with a relevant decrease in CL (up to 20.1-fold) among 72 healthy volunteers treated with cobicistat, owing to strong inhibitory activity on CYP3A4. Coadministration of midazolam and a strong inhibitor of CYP3A4 should be avoided, or intravenous administration of midazolam with cobicistat should be only considered in the ICU setting where close monitoring of respiratory function should be performed [69].
Several PK studies investigated the impact of ritonavir and/or of ritonavir-boosted PIs on benzodiazepine metabolism and exposure [90-95]. Greenblatt et al. [90] found a significant increase in alprazolam exposure (2.48-fold in AUC) in ten healthy volunteers concomitantly receiving ritonavir, leading to augmented sedation and performance impairment. Similarly, Menon et al. [91] found a moderate increase in alprazolam disposition ( $34 \%$ in AUC) in 12 healthy subjects receiving paritaprevir/ritonavir, due to inhibitory activity of ritonavir on CYP3A4. In the same study [91], authors also reported no significant variations in zolpidem exposure in 12 healthy volunteers with concomitant administration of paritaprevir/ritonavir. A significant increase in midazolam exposure (respectively from 3.02-fold to 26.41-fold for AUC and from 1.15-fold to 4.27 -fold for $C_{\max }$ ) in healthy volunteers concomitantly treated with ritonavir alone or ritonavir-boosted PIs has been reported, caused by strong inhibition of CYP3A4 [92-95]. Notably, a more pronounced increase in midazolam disposition was found with oral compared with intravenous administration [95]. The concomitant use of midazolam and ritonavir should be avoided, or solely reserved for intubated ICU patients in which lower midazolam dosage should be required to achieve adequate sedation [69]. A retrospective cohort study [96] including 241 patients with HIV undergoing bronchoscopy reported an increased relative risk of severe prolonged sedation (risk ratio (RR) 6.21 ; 95\% confidence interval 1.53-25.12) in patients receiving intravenous midazolam (median dose 4 $\mathrm{mg}$ ) concomitantly treated with ritonavir-boosted PIs compared with those using other antiretroviral agents. Greenblatt et al. [97] found a significant increase in triazolam exposure (20.39-fold in AUC) in six healthy volunteers receiving ritonavir, due to strong inhibitory activity on CYP3A4 and resulting in increased sedation and impairment of psychomotor performance. Similarly, Culm-Merdeck et al. [98] reported a relevant increase in triazolam disposition (21.1fold in AUC) in 23 healthy subjects concomitantly treated with ritonavir. Consequently, coadministration of triazolam and ritonavir-boosted PIs should be avoided to prevent excessive sedation and respiratory depression. Conversely, ritonavir caused only a moderate increase in zolpidem exposure (respectively 1.28-fold and 1.22-fold in AUC and $C_{\max }$ ) [97], thus

A summary of predicted PK and PD interactions between sedative hypnotics and COVID-19 agents is shown in Table 4 of the ESM. A high or moderate risk of clinically relevant drug interactions may be expected only when sedative hypnotics that are substrates of CYP3A4 are concomitantly used in patients with COVID-19 receiving antiretroviral agents that are strong CYP3A4 inhibitors, namely ritonavir or cobicistat. The combination may potentially cause severe prolonged sedation associated with respiratory depression (particularly with midazolam and triazolam). In 
this scenario, the use of oxazepam-like benzodiazepines (namely lorazepam, lormetazepam, oxazepam and temazepam), exhibiting no metabolism through CYP450 pathway, should be preferred [99]. According to the study of Zhuang et al. [87], a higher midazolam dose could be required to provide adequate sedation in patients with severe COVID19 affected by a cytokine storm and managed with anti-IL-6 agents (tocilizumab or siltuximab).No clinically relevant interactions involving remdesivir, colchicine, dexamethasone, enoxaparin, ruxolitinib, bamlanivimab and casirivimab/imdevimab are expected to occur with sedative hypnotics according to PK/PD properties.

Overall, according to real-world evidence and PK/PD properties, sedative hypnotics metabolised by CYP3A4 pose the major concern for the occurrence of clinically relevant interactions with lopinavir/ritonavir and darunavir/ cobicistat. Concomitant administration of midazolam and triazolam with PIs should be avoided in non-ICU patients. Oxazepam-like benzodiazepines (i.e. lorazepam, lormetazepam, oxazepam and temazepam) should be preferred as first-line therapy for the management of anxiety disorders by virtue of a favourable PK/PD profile.

\section{Conclusions and Perspectives}

Overall, real-life experiences of clinically relevant drug interactions between psychotropic medications and repurposed COVID-19 agents occurring in patients with severe acute respiratory syndrome coronavirus 2 infection are scarce. Only two case reports describing the onset of serotonin syndrome in patients with COVID-19 cotreated with lopinavir/ritonavir and duloxetine, risperidone or lithium exist [48]. Unfortunately, no PK analysis or TDM was performed in these cases to prove the increased exposure of psychotropic agents resulting from inhibitory activity on CYP450 exhibited by ritonavir.

The PK studies that investigated the impact of repurposed COVID-19 agents on psychotropic medications in the majority of cases were performed among healthy subjects (Tables 3, 4, 5, 6). Accordingly, translation of these findings to patients with severe COVID-19 may be questionable, considering that the magnitude of the impact of DDIs due to multi-organ failure and/or to cytokine storm often occurring in patients with COVID-19 could not be adequately addressed [12]. Nevertheless, the available studies provide essential findings to prioritise the risk of clinically relevant drug interactions in patients with COVID-19, calling for avoidance of certain associations (e.g. midazolam/triazolam with lopinavir/ritonavir [92-95, 97, 98]), for some dose adjustments (e.g. lamotrigine or carbamazepine when lopinavir/ritonavir is used [72-79]) or for some psychotropic replacement (e.g. olanzapine instead of quetiapine in case of concomitant use of lopinavir/ritonavir or darunavir/cobicistat $[58,59])$.

According to PK/PD properties and available evidence, antiretrovirals containing strong inhibitors of CYP450 (lopinavir/ritonavir and darunavir/cobicistat) pose the major issues in terms of potential occurrence of clinically relevant drug interactions with several antipsychotic agents. This represents an important concern given that lopinavir/ritonavir and darunavir/cobicistat were used in mild-to-moderate forms of COVID-19 (also including outpatient management), in which long-term concomitant treatment with oral psychotropic agents are usually maintained. In contrast, in patients with severe COVID-19 requiring ICU admission, long-term therapies (including psychotropic agents) are abruptly suspended, and thus the risk should be lower in this setting. In this latter scenario, only a few drugs are commonly used through parenteral administration to provide adequate sedation or a delirolytic effect (e.g. midazolam, haloperidol), and potential relevant drug interactions could be successfully managed thanks to the fact that ICU patients are intubated (i.e. no risk of severe respiratory depression caused by midazolam overexposure) and carefully monitored (i.e. early identification of QT prolongation caused by increased disposition of haloperidol). Indeed, some patients may receive long-acting injectable antipsychotic agents to manage schizophrenia or bipolar disorder. In this scenario, clinically relevant drug interactions may also occur despite ICU admission because of the long elimination half-life of these agents.

Notably, the use of lopinavir/ritonavir and darunavir/ cobicistat in COVID-19 is currently limited, owing to poor evidence of efficacy in severe acute respiratory syndrome coronavirus 2 infection [100, 101], and this decreases considerably the risk of clinically significant interactions. A practical algorithm to manage the occurrence of clinically relevant drug interactions between psychotropic medications and COVID-19 agents according to disease severity is provided in Fig. 2. In outpatient/inpatient scenarios concerning mild-to-moderate COVID-19, antiretrovirals (lopinavir/ritonavir and darunavir/cobicistat) represent the agents showing the higher risk of significant drug interactions. While in the case of concomitant administration with antidepressants, only a moderate risk of clinically relevant interactions could be recognised according to an increase in antidepressant exposure and QT prolongation (e.g. citalopram, fluoxetine, TCAs, trazodone), a high risk of significant interactions resulting in life-threatening events could be expected with several antipsychotic agents (i.e. haloperidol, quetiapine), mood stabilisers (i.e. carbamazepine) and sedative hypnotics (i.e., midazolam, triazolam). Notably, the dosage of different psychotropic agents (e.g. bupropion, olanzapine, lamotrigine) should be increased in the case of concomitant 
administration with antiretrovirals to achieve desired effects. In this scenario, the use of psychotropic agents (e.g. oxazepam-like benzodiazepines, olanzapine), exhibiting no or minimal metabolism through the CYP450 pathway (particularly CYP3A4), should be preferred. Conversely, in inpatient/ICU scenarios concerning moderate-to-severe COVID19 , no concerns for the occurrence of clinically relevant interactions with psychotropic agents exist, given that oral medications are usually abruptly suspended. Anti-inflammatory therapies used for severe COVID-19 pose no relevant PK/PD issues able to cause high-risk drug interactions with psychotropic agents. Only the use of anti-IL-1 or IL-6 agents may reverse the inhibitory effect of pro-inflammatory cytokines on CYP450, thus potentially causing underexposure of midazolam or haloperidol, while carbamazepine may reduce the disposition of different COVID-19 therapies (e.g. colchicine, dexamethasone, ruxolitinib).

Interestingly, Anmella et al. [102] provided practical recommendations for patients affected by psychiatric disorders requiring concomitant treatment with lopinavir/ritonavir in the COVID-19 setting. Electrocardiogram monitoring was recommended for properly managing expected
PK/PD interactions, leading to QT prolongation and potential life-threatening arrhythmias. Additionally, three different clinical scenarios were discussed. First, olanzapine was recommended as first-line therapy in agitated delirium, and quetiapine should be avoided. This is consistent with the findings reported in PK studies and in case reports [58, 59, 62-64, 71]. Second, essential treatments (e.g. antipsychotic agents, mood stabilisers) should be maintained in severe mental illness and should be prioritised, avoiding abrupt withdrawal. In this scenario, careful monitoring for clinically relevant PK/PD interactions coupled with dose adjustment should be implemented. This is especially true when psychotropic agents at high risk for drug interactions are co-administered with lopinavir/ritonavir. Third, in nonsevere mental illness with depressive/anxiety symptoms, psychological support coupled with prompt identification and treatment of symptoms should be provided. In this scenario, the use of antidepressants or sedative hypnotics showing a low risk for PK/PD interactions with COVID-19 agents (e.g. oxazepam-like benzodiazepines, fluoxetine, sertraline) should be preferred. This is consistent with the findings reported in PK studies [44, 45]. It should not be

\begin{tabular}{|c|c|}
\hline $\begin{array}{l}\text { MILD-MODERATE COVID-19 } \\
\text { (Antivirals } \gg \text { Anti-inflammatory) }\end{array}$ & $\begin{array}{l}\text { MODERATE-SEVERE COVID-19 } \\
\text { (Anti-inflammatory } \gg \text { Antivirals) }\end{array}$ \\
\hline
\end{tabular}

\begin{tabular}{|c|c|c|}
\hline \multicolumn{3}{|c|}{ Clinically relevant drug interactions with psychotropics? } \\
\hline $\begin{array}{l}\text { QT prolongation and increased } \\
\text { exposure of several antidepressants } \\
\text { with antiretrovirals }\end{array}$ & Antidepressants & $\begin{array}{l}\text { Oral antidepressant usually } \\
\text { stopped in ICU patients. } \\
\text { No risk of relevant DDIs }\end{array}$ \\
\hline $\begin{array}{l}\text { Coadministration of haloperidol or } \\
\text { quetiapine with antiretrovirals } \\
\text { should be avoided }\end{array}$ & Antipsychotics & $\begin{array}{c}\text { Consider increased dose of } \\
\text { parenteral haloperidol when } \\
\text { anti-IL-1/6 are used }\end{array}$ \\
\hline $\begin{array}{l}\text { Coadministration of carbamazepine } \\
\text { with antiretrovirals should be } \\
\text { avoided (consider TDM) }\end{array}$ & Mood stabilizers & $\begin{array}{l}\text { Relevant underexposure of } \\
\text { different COVID-19 agents when } \\
\text { carbamazepine is used }\end{array}$ \\
\hline $\begin{array}{l}\text { Coadministration of midazolam or } \\
\text { triazolam with antiretrovirals } \\
\text { should be avoided }\end{array}$ & Sedative-hypnotics & $\begin{array}{l}\text { Consider increased dose of } \\
\text { intravenous midazolam when } \\
\text { anti-IL- } 1 / 6 \text { are used }\end{array}$ \\
\hline
\end{tabular}

Fig. 2 Proposed algorithm for the management of potential clinically relevant interactions between psychotropic medications and repurposed COVID-19 agents according to disease severity/stage of the infection. $B Z D$ benzodiazepine, $D D I s$ drug-drug interactions, $I C U$ intensive care unit, $I L$ interleukin, $T D M$ therapeutic drug monitoring 
overlooked that close monitoring should be performed whenever dose adjustments are extended beyond the recommendations provided in the summary of product characteristics of a specific agent. Furthermore, in the case of PD interactions leading to QT prolongation or other additive toxicity (e.g. hepatic, haematological), tailored monitoring should be promptly implemented.

Notably, the potential interaction profile of remdesivir is not fully understood, given that only in vitro evidence with unclear clinical implications is available, and no in vivo drug interaction study has been performed or cases of relevant interactions described [103]. In vitro studies demonstrated that remdesivir could exhibit moderate or weak inhibitory activity respectively on CYP3A4 or CYP1A2, CYP2C9, CYP2C19, and CYP2D6 isozymes, and a relevant inhibitory effect on carboxylesterase-2 through covalent modifications has been reported [103, 104]. However, the clinical relevance of these findings is questionable considering that remdesivir is a pro-drug that is rapidly degraded in vivo, and therefore the potential for clinically significant drug interactions is likely to be limited. Administration of sedative hypnotics that are substrates of CYP3A4 2 hours after a remdesivir infusion has been recommended to avoid potentially relevant interactions in the case of cotreatment [29].

In conclusion, clinically relevant drug interactions between psychotropic medications and COVID-19 agents mainly concern antiretrovirals. The poor efficacy showed by lopinavir/ritonavir and/or darunavir/cobicistat in COVID-19 prospective clinical trials to date has led to a decrease in their use. Therefore, the risk of occurrence of relevant interactions is currently quite limited. The major issue that is still to be addressed is represented by the impact that a DDI may have on psychotropic agents in patients with moderate-tosevere COVID-19. Multi-organ failure and a cytokine storm occurring in the late stage of COVID-19 infections might be responsible of high-risk DDIs with psychotropic medications whose magnitude and clinical relevance unfortunately are still to be defined.

Supplementary Information The online version contains supplementary material available at https://doi.org/10.1007/s40263-021-00811-2.

\section{Declarations}

Funding Open access funding provided by Alma Mater Studiorum Università di Bologna within the CRUI-CARE Agreement. No funding was used to assist with the preparation of this review.

Conflict of interest Federico Pea participated in the speaker bureau for Angelini, Basilea Pharmaceutica Gilead, Hikma, Merck Sharp and Dohme, Nordic Pharma, Pfizer and Sanofi Aventis, and in an advisory board for Angelini, Basilea Pharmaceutica, Correvio, Gilead, Merck Sharp and Dohme, Nordic Pharma, Novartis, Pfizer and Thermo-Fisher. Milo Gatti and Fabrizio De Ponti have no conflicts of interest that are directly relevant to the content of this article.
Ethics approval Not applicable.

Consent to participate Not applicable.

Consent for publication Not applicable.

Availability of data and material Not applicable.

Code availability Not applicable.

Author contributions MG and FP made substantial contributions to the conception of the manuscript. MG was involved in drafting the manuscript. FDP and FP made substantial contributions in critically revising the manuscript for important intellectual content. All the authors approved the final version of the manuscript.

Open Access This article is licensed under a Creative Commons Attribution-NonCommercial 4.0 International License, which permits any non-commercial use, sharing, adaptation, distribution and reproduction in any medium or format, as long as you give appropriate credit to the original author(s) and the source, provide a link to the Creative Commons licence, and indicate if changes were made. The images or other third party material in this article are included in the article's Creative Commons licence, unless indicated otherwise in a credit line to the material. If material is not included in the article's Creative Commons licence and your intended use is not permitted by statutory regulation or exceeds the permitted use, you will need to obtain permission directly from the copyright holder. To view a copy of this licence, visit http://creativecommons.org/licenses/by-nc/4.0/.

\section{References}

1. Zhou P, Yang XL, Wang XG, Hu B, Zhang L, Zhang W, et al. A pneumonia outbreak associated with a new coronavirus of probable bat origin. Nature. 2020;579(7798):270-3.

2. WHO. WHO Director-General's remarks at the media briefing on 2019-nCoV on 11 February 2020. Available from: https:// www.who.int/dg/speeches/detail/who-director-general-s-remar ks-at-the-media-briefing-on-2019-ncov-on-11-february-2020. Accessed 22 Dec 2020.

3. WHO. Virtual press conference on COVID-19, 11 March 2020. Available from: https://www.euro.who.int/en/health-topics/healthemergencies/coronavirus-covid19/news/news/2020/3/who-annou nces-covid-19-outbreak-a-pandemic. Accessed 22 Dec 2020.

4. European Centre for Disease Prevention and Control. COVID19 situation update worldwide. Available from: https://www. ecdc.europa.eu/en/geographical-distribution-2019-ncov-cases. Accessed 22 Dec 2020.

5. Siddiqi HK, Mehra MR. COVID-19 illness in native and immunosuppressed states: a clinical-therapeutic staging proposal. J Heart Lung Transplant. 2020;39(5):405-7.

6. European Medicines Agency. First COVID-19 treatment recommended for EU authorisation, 25 June 2020. Available from: https://www.ema.europa.eu/en/documents/press-release/firstcovid-19-treatment-recommended-eu-authorisation_en.pdf. Accessed 22 Dec 2020.

7. US Food and Drug Administration. FDA's approval of Veklury (remdesivir) for the treatment of COVID-19: the science of safety and effectiveness, 22 October 2020. Available from: https://www. fda.gov/drugs/drug-safety-and-availability/fdas-approval-veklu ry-remdesivir-treatment-covid-19-science-safety-and-effectiven ess. Accessed 22 Dec 2020. 
8. Mahase E. Covid-19: FDA authorises neutralising antibody bamlanivimab for non-admitted patients. BMJ. 2020;371:m4362.

9. Tuccori M, Ferraro S, Convertino I, Cappello E, Valdiserra G, Blandizzi C, et al. Anti-SARS-CoV-2 neutralizing monoclonal antibodies: clinical pipeline. MAbs. 2020;12(1):1854149.

10. Gatti M, Raschi E, Poluzzi E, Martignani C, Salvagni S, Ardizzoni A, et al. The complex management of atrial fibrillation and cancer in the COVID-19 era: drug interactions, thromboembolic risk, and proarrhythmia. Curr Heart Fail Rep. 2020;17(6):365-83.

11. Hodge C, Marra F, Marzolini C, Boyle A, Gibbons S, Siccardi $\mathrm{M}$, et al. Drug interactions: a review of the unseen danger of experimental COVID-19 therapies. J Antimicrob Chemother. 2020;75(12):3417-24.

12. Zeitlinger M, Koch BCP, Bruggemann R, De Cock P, Felton T, Hites M, PK/PD of Anti-Infectives Study Group (EPASG) of the European Society of Clinical Microbiology, Infectious Diseases (ESCMID), et al. Pharmacokinetics/pharmacodynamics of antiviral agents used to treat SARS-CoV-2 and their potential interaction with drugs and other supportive measures: a comprehensive review by the PK/PD of Anti-Infectives Study Group of the European Society of Antimicrobial Agents. Clin Pharmacokinet. 2020;59(10):1195-216.

13. Lemaitre F, Solas C, Grégoire M, Lagarce L, Elens L, Polard E, French Society of Pharmacology, Therapeutics (SFPT), the International Association of Therapeutic Drug Monitoring, Clinical Toxicology (IATDMCT), et al. Potential drug-drug interactions associated with drugs currently proposed for COVID-19 treatment in patients receiving other treatments. Fundam Clin Pharmacol. 2020;34(5):530-47.

14. Zhang K, Zhou X, Liu H, Hashimoto K. Treatment concerns for psychiatric symptoms in patients with COVID-19 with or without psychiatric disorders. Br J Psychiatry. 2020;217(1):351.

15. Steardo L Jr, Steardo L, Verkhratsky A. Psychiatric face of COVID-19. Transl Psychiatry. 2020;10(1):261.

16. Vindegaard N, Benros ME. COVID-19 pandemic and mental health consequences: systematic review of the current evidence. Brain Behav Immun. 2020;89:531-42.

17. Rogers JP, Chesney E, Oliver D, Pollak TA, McGuire P, FusarPoli P, et al. Psychiatric and neuropsychiatric presentations associated with severe coronavirus infections: a systematic review and meta-analysis with comparison to the COVID-19 pandemic. Lancet Psychiatry. 2020;7(7):611-27.

18. Dubey S, Biswas P, Ghosh R, Chatterjee S, Dubey MJ, Chatterjee $\mathrm{S}$, et al. Psychosocial impact of COVID-19. Diabetes Metab Syndr. 2020;14(5):779-88.

19. Torales J, O'Higgins M, Castaldelli-Maia JM, Ventriglio A. The outbreak of COVID-19 coronavirus and its impact on global mental health. Int J Soc Psychiatry. 2020;66(4):317-20.

20. Ostuzzi G, Papola D, Gastaldon C, Schoretsanitis G, Bertolini F, Amaddeo F, et al. Safety of psychotropic medications in people with COVID-19: evidence review and practical recommendations. BMC Med. 2020;18(1):215.

21. Bilbul M, Paparone P, Kim AM, Mutalik S, Ernst CL. Psychopharmacology of COVID-19. Psychosomatics. 2020;61(5):411-27.

22. Bishara D, Kalafatis C, Taylor D. Emerging and experimental treatments for COVID-19 and drug interactions with psychotropic agents. Ther Adv Psychopharmacol. 2020;10:2045125320935306

23. Lenoir C, Daali Y, Rollason V, Curtin F, Gloor Y, Bosilkovska $\mathrm{M}$, et al. Impact of acute inflammation on cytochromes P450 activity assessed by the Geneva Cocktail. Clin Pharmacol Ther. 2020. https://doi.org/10.1002/cpt.2146.

24. Fajgenbaum DC, June CH. Cytokine storm. N Engl J Med. 2020;383(23):2255-73.
25. La MK, Thompson Bastin ML, Gisewhite JT, Johnson CA, Flannery AH. Impact of restarting home neuropsychiatric medications on sedation outcomes in medical intensive care unit patients. J Crit Care. 2018;43:102-7.

26. Smolders EJ, de Kanter CT, de Knegt RJ, van der Valk M, Drenth JP, Burger DM. Drug-drug interactions between directacting antivirals and psychoactive medications. Clin Pharmacokinet. 2016;55(12):1471-94.

27. Woosley RL, Heise CW, Romero KA. QTdrugs List. Accession date. Oro Valley (AZ): AZCERT, Inc. Available from: www. Crediblemeds.org. Accessed 28 Dec 2020.

28. European Medicines Agency. Guideline on the investigation of drug interactions. 2013. Available from: https://www.ema. europa.eu/en/investigation-drug-interactions. Accessed $28 \mathrm{Dec}$ 2020.

29. Liverpool Drug Interaction Group. COVID-19 drug interactions. Available from: https://www.covid19-druginteractions. org/. Accessed 28 Dec 2020.

30. Pea F, Furlanut M. Pharmacokinetic aspects of treating infections in the intensive care unit: focus on drug interactions. Clin Pharmacokinet. 2001;40(11):833-68.

31. Akamine Y, Yasui-Furukori N, Ieiri I, Uno T. Psychotropic drug-drug interactions involving P-glycoprotein. CNS Drugs. 2012;26(11):959-73.

32. Preskorn SH. Drug-drug interactions (DDIs) in psychiatric practice, part 9: interactions mediated by drug-metabolizing cytochrome P450 enzymes. J Psychiatr Pract. 2020;26(2):126-34

33. Anson BD, Weaver JG, Ackerman MJ, Akinsete O, Henry K, January CT, et al. Blockade of HERG channels by HIV protease inhibitors. Lancet. 2005;365(9460):682-6.

34. Ye Q, Wang B, Mao J. The pathogenesis and treatment of the 'cytokine storm' in COVID-19. J Infect. 2020;80(6):607-13.

35. Kim S, Östör AJ, Nisar MK. Interleukin-6 and cytochrome-P450, reason for concern? Rheumatol Int. 2012;32(9):2601-4.

36. Aitken AE, Morgan ET. Gene-specific effects of inflammatory cytokines on cytochrome P450 2C, 2B6 and 3A4 mRNA levels in human hepatocytes. Drug Metab Dispos. 2007;35(9):1687-93.

37. Frye RF, Schneider VM, Frye CS, Feldman AM. Plasma levels of TNF-alpha and IL-6 are inversely related to cytochrome P450-dependent drug metabolism in patients with congestive heart failure. J Card Fail. 2002;8(5):315-9.

38. Cojutti PG, Londero A, Della Siega P, Givone F, Fabris M, Biasizzo J, et al. Comparative population pharmacokinetics of darunavir in SARS-CoV-2 patients vs. HIV patients: the role of interleukin-6. Clin Pharmacokinet. 2020;59(10):1251-60.

39. Preskorn SH, Quadri S. Why are patients with COVID-19 at risk for drug-drug interactions? J Psychiatr Pract. 2020;26(6):485-92.

40. Milosavljevic F, Bukvic N, Pavlovic Z, Miljevic C, Pešic V, Molden E, et al. Association of CYP2C19 and CYP2D6 poor and intermediate metabolizer status with antidepressant and antipsychotic exposure: a systematic review and meta-analysis. JAMA Psychiat. 2020. https://doi.org/10.1001/jamapsychiatry. 2020.3643.

41. Gutierrez MM, Rosenberg J, Abramowitz W. An evaluation of the potential for pharmacokinetic interaction between escitalopram and the cytochrome P450 3A4 inhibitor ritonavir. Clin Ther. 2003;25(4):1200-10.

42. Lorenzini KI, Calmy A, Ambrosioni J, Assouline B, Daali Y, Fathi M, et al. Serotonin syndrome following drug-drug interactions and CYP2D6 and CYP2C19 genetic polymorphisms in an HIV-infected patient. AIDS. 2012;26(18):2417-8.

43. van der Lee MJ, Blenke AA, Rongen GA, Verwey-van Wissen $\mathrm{CP}$, Koopmans PP, Pharo C, et al. Interaction study of the combined use of paroxetine and fosamprenavir-ritonavir in healthy subjects. Antimicrob Agents Chemother. 2007;51(11):4098-104. 
44. Hanan NJ, Paul ME, Huo Y, Kapetanovic S, Smith E, Siberry G, et al. Sertraline Pharmacokinetics in HIV-infected and uninfected children, adolescents, and young adults. Front Pediatr. 2019;7:16.

45. Ouellet D, Hsu A, Qian J, Lamm JE, Cavanaugh JH, Leonard $\mathrm{JM}$, et al. Effect of fluoxetine on pharmacokinetics of ritonavir. Antimicrob Agents Chemother. 1998;42(12):3107-12.

46. Samuel NG, Seifert CF. Risk of bleeding in patients on full-dose enoxaparin with venous thromboembolism and selective serotonin reuptake inhibitors. Ann Pharmacother. 2017;51(3):226-31.

47. Laporte S, Chapelle C, Caillet P, Beyens MN, Bellet F, Delavenne $\mathrm{X}$, et al. Bleeding risk under selective serotonin reuptake inhibitor (SSRI) antidepressants: a meta-analysis of observational studies. Pharmacol Res. 2017;118:19-32.

48. Mas Serrano M, Pérez-Sánchez JR, Portela Sánchez S, De La Casa-Fages B, Mato Jimeno V, Pérez Tamayo I, et al. Serotonin syndrome in two COVID-19 patients treated with lopinavir/ritonavir. J Neurol Sci. 2020;415:116944.

49. Mascolini M, Mellors JW, Richman DD, Boucher CA, Larder BA. HIV and hepatitis virus resistance to antivirals: review of data from the XIX International HIV and Hepatitis Virus Drug Resistance Workshop and curative strategies. Antivir Ther. 2011;16(2):263-86.

50. Aarnoutse RE, Kleinnijenhuis J, Koopmans PP, Touw DJ, Wieling J, Hekster YA, et al. Effect of low-dose ritonavir (100 mg twice daily) on the activity of cytochrome P450 2D6 in healthy volunteers. Clin Pharmacol Ther. 2005;78(6):664-74.

51. Cleare A, Pariante CM, Young AH, Anderson IM, Christmas D, Cowen PJ, Members of the Consensus Meeting, et al. Evidence-based guidelines for treating depressive disorders with antidepressants: a revision of the 2008 British Association for Psychopharmacology guidelines. J Psychopharmacol. 2015;29(5):459-525.

52. Park J, Vousden M, Brittain C, McConn DJ, Iavarone L, Ascher J, et al. Dose-related reduction in bupropion plasma concentrations by ritonavir. J Clin Pharmacol. 2010;50(10):1180-7.

53. Hogeland GW, Swindells S, McNabb JC, Kashuba AD, Yee GC, Lindley CM. Lopinavir/ritonavir reduces bupropion plasma concentrations in healthy subjects. Clin Pharmacol Ther. 2007;81(1):69-75.

54. Kharasch ED, Mitchell D, Coles R, Blanco R. Rapid clinical induction of hepatic cytochrome $\mathrm{P} 4502 \mathrm{~B} 6$ activity by ritonavir. Antimicrob Agents Chemother. 2008;52(5):1663-9.

55. Hesse LM, Greenblatt DJ, von Moltke LL, Court MH. Ritonavir has minimal impact on the pharmacokinetic disposition of a single dose of bupropion administered to human volunteers. J Clin Pharmacol. 2006;46(5):567-76.

56. Greenblatt DJ, von Moltke LL, Harmatz JS, Fogelman SM, Chen G, Graf JA, et al. Short-term exposure to low-dose ritonavir impairs clearance and enhances adverse effects of trazodone. $\mathrm{J}$ Clin Pharmacol. 2003;43(4):414-22.

57. Ferri N, Bellosta S, Baldessin L, Boccia D, Racagni G, Corsini A. Pharmacokinetics interactions of monoclonal antibodies. Pharmacol Res. 2016;111:592-9.

58. Penzak SR, Hon YY, Lawhorn WD, Shirley KL, Spratlin V, Jann MW. Influence of ritonavir on olanzapine pharmacokinetics in healthy volunteers. J Clin Psychopharmacol. 2002;22(4):366-70.

59. Jacobs BS, Colbers AP, Velthoven-Graafland K, Schouwenberg BJ, Burger DM. Effect of fosamprenavir/ritonavir on the pharmacokinetics of single-dose olanzapine in healthy volunteers. Int J Antimicrob Agents. 2014;44(2):173-7.

60. Aung GL, O’Brien JG, Tien PG, Kawamoto LS. Increased aripiprazole concentrations in an HIV-positive male concurrently taking duloxetine, darunavir, and ritonavir. Ann Pharmacother. 2010;44(11):1850-4.
61. Hahn M, Roll SC. Dosing recommendations of aripiprazole depot with strong cytochrome P450 3A4 inhibitors: a relapse risk. Drug Saf Case Rep. 2016;3(1):5.

62. Pollack TM, McCoy C, Stead W. Clinically significant adverse events from a drug interaction between quetiapine and atazanavir-ritonavir in two patients. Pharmacotherapy. 2009;29(11):1386-91.

63. Geraci MJ, McCoy SL, Crum PM, Patel RA. Antipsychoticinduced priapism in an HIV patient: a cytochrome P450-mediated drug interaction. Int J Emerg Med. 2010;3(2):81-4.

64. Hill L, Lee KC. Pharmacotherapy considerations in patients with HIV and psychiatric disorders: focus on antidepressants and antipsychotics. Ann Pharmacother. 2013;47(1):75-89.

65. Kelly DV, Béique LC, Bowmer MI. Extrapyramidal symptoms with ritonavir/indinavir plus risperidone. Ann Pharmacother. 2002;36(5):827-30.

66. Jover F, Cuadrado JM, Andreu L, Merino J. Reversible coma caused by risperidone-ritonavir interaction. Clin Neuropharmacol. 2002;25(5):251-3.

67. Gonzalez LS, Kothari K, Kasle DA. Three cases of late onset angioedema in nursing home human immunodeficiency virus patients on ritonavir and risperidone. J Clin Psychopharmacol. 2016;36(1):95-7.

68. Lee SI, Klesmer J, Hirsch BE. Neuroleptic malignant syndrome associated with use of risperidone, ritonavir, and indinavir: a case report. Psychosomatics. 2000;41(5):453-4.

69. Goodlet KJ, Zmarlicka MT, Peckham AM. Drug-drug interactions and clinical considerations with co-administration of antiretrovirals and psychotropic drugs. CNS Spectr. 2019;24(3):287-312.

70. Repetto MJ, Petitto JM. Psychopharmacology in HIV-infected patients. Psychosom Med. 2008;70(5):585-92.

71. Sampson MR, Cao KY, Gish PL, Hyon K, Mishra P, Tauber $\mathrm{W}$, et al. Dosing recommendations for quetiapine when coadministered with HIV protease inhibitors. J Clin Pharmacol. 2019;59(4):500-9.

72. Burger DM, Huisman A, Van Ewijk N, Neisingh H, Van Uden $\mathrm{P}$, Rongen GA, et al. The effect of atazanavir and atazanavir/ ritonavir on UDP-glucuronosyltransferase using lamotrigine as a phenotypic probe. Clin Pharmacol Ther. 2008;84(6):698-703.

73. van der Lee MJ, Dawood L, ter Hofstede HJ, de Graaff-Teulen MJ, van Ewijk-Beneken Kolmer EW, Caliskan-Yassen N. Lopinavir/ritonavir reduces lamotrigine plasma concentrations in healthy subjects. Clin Pharmacol Ther. 2006;80(2):159-68.

74. Mateu-de Antonio J, Grau S, Gimeno-Bayón JL, Carmona A. Ritonavir-induced carbamazepine toxicity. Ann Pharmacother. 2001;35(1):125-6.

75. Bates DE, Herman RJ. Carbamazepine toxicity induced by lopinavir/ritonavir and nelfinavir. Ann Pharmacother. 2006;40(6):1190-5.

76. Berbel Garcia A, Latorre Ibarra A, Porta Etessam J, Martinez Salio A, Perez Martinez D, Siaz Diaz R, et al. Protease inhibitor-induced carbamazepine toxicity. Clin Neuropharmacol. 2000;23(4):216-8.

77. Kato Y, Fujii T, Mizoguchi N, Takata N, Ueda K, Feldman MD, Kayser SR. Potential interaction between ritonavir and carbamazepine. Pharmacotherapy. 2000;20(7):851-4.

78. Burman W, Orr L. Carbamazepine toxicity after starting combination antiretroviral therapy including ritonavir and efavirenz. AIDS. 2000;14(17):2793-4.

79. DiCenzo R, Peterson DR, Cruttenden K, Mariuz P, Rezk NL, Hochreiter J, et al. Effects of minocycline and valproic acid coadministration on atazanavir plasma concentrations in human immunodeficiency virus-infected adults receiving atazanavir-ritonavir. Antimicrob Agents Chemother. 2008;52(9):3035-9. 
80. Sheehan NL, Brouillette MJ, Delisle MS, Allan J. Possible interaction between lopinavir/ritonavir and valproic acid exacerbates bipolar disorder. Ann Pharmacother. 2006;40(1):147-50.

81. Birbeck GL, French JA, Perucca E, Simpson DM, Fraimow H, George JM, Quality Standards Subcommittee of the American Academy of Neurology; Ad Hoc Task Force of the Commission on Therapeutic Strategies of the International League Against Epilepsy, et al. Evidence-based guideline: antiepileptic drug selection for people with HIV/AIDS: report of the Quality Standards Subcommittee of the American Academy of Neurology and the Ad Hoc Task Force of the Commission on Therapeutic Strategies of the International League Against Epilepsy. Neurology. 2012;78(2):139-45.

82. Buoli M, Serati M, Botturi A, Altamura AC. The risk of thrombocytopenia during valproic acid therapy: a critical summary of available clinical data. Drugs R D. 2018;18(1):1-5.

83. Finley PR. Drug interactions with lithium: an update. Clin Pharmacokinet. 2016;55(8):925-41.

84. Spina E, Pisani F, Perucca E. Clinically significant pharmacokinetic drug interactions with carbamazepine: an update. Clin Pharmacokinet. 1996;31(3):198-214.

85. Villikka K, Kivistö KT, Neuvonen PJ. The effect of dexamethasone on the pharmacokinetics of triazolam. Pharmacol Toxicol. 1998;83(3):135-8.

86. Czock D, Keller F, Rasche FM, Häussler U. Pharmacokinetics and pharmacodynamics of systemically administered glucocorticoids. Clin Pharmacokinet. 2005;44(1):61-98.

87. Zhuang Y, de Vries DE, Xu Z, Marciniak SJ Jr, Chen D, Leon F, Davis HM, et al. Evaluation of disease-mediated therapeutic protein-drug interactions between an anti-interleukin- 6 monoclonal antibody (sirukumab) and cytochrome P450 activities in a phase 1 study in patients with rheumatoid arthritis using a cocktail approach. J Clin Pharmacol. 2015;55(12):1386-94.

88. Machavaram KK, Endo-Tsukude C, Terao K, Gill KL, Hatley OJ, Gardner I, et al. Simulating the impact of elevated levels of interleukin- 6 on the pharmacokinetics of various CYP450 substrates in patients with neuromyelitis optica or neuromyelitis optica spectrum disorders in different ethnic populations. AAPS J. 2019;21(3):42.

89. Mathias AA, German P, Murray BP, Wei L, Jain A, West S, et al. Pharmacokinetics and pharmacodynamics of GS-9350: a novel pharmacokinetic enhancer without anti-HIV activity. Clin Pharmacol Ther. 2010;87(3):322-9.

90. Greenblatt DJ, von Moltke LL, Harmatz JS, Durol AL, Daily JP, Graf JA, et al. Alprazolam-ritonavir interaction: implications for product labeling. Clin Pharmacol Ther. 2000;67(4):335-41.

91. Menon RM, Badri PS, Wang T, Polepally AR, Zha J, Khatri A, et al. Drug-drug interaction profile of the all-oral anti-hepatitis $\mathrm{C}$ virus regimen of paritaprevir/ritonavir, ombitasvir, and dasabuvir. J Hepatol. 2015;63(1):20-9.
92. Schmitt C, Hofmann C, Riek M, Patel A, Zwanziger E. Effect of saquinavir-ritonavir on cytochrome P450 3A4 activity in healthy volunteers using midazolam as a probe. Pharmacotherapy. 2009;29(10):1175-81.

93. Greenblatt DJ, Peters DE, Oleson LE, Harmatz JS, MacNab MW, Berkowitz N, et al. Inhibition of oral midazolam clearance by boosting doses of ritonavir, and by 4,4-dimethyl-benziso-(2H)selenazine (ALT-2074), an experimental catalytic mimic of glutathione oxidase. Br J Clin Pharmacol. 2009;68(6):920-7.

94. Eichbaum C, Cortese M, Blank A, Burhenne J, Mikus G. Concentration effect relationship of CYP3A inhibition by ritonavir in humans. Eur J Clin Pharmacol. 2013;69(10):1795-800.

95. Hafner V, Jäger M, Matthée AK, Ding R, Burhenne J, Haefeli WE, et al. Effect of simultaneous induction and inhibition of CYP3A by St John's Wort and ritonavir on CYP3A activity. Clin Pharmacol Ther. 2010;87(2):191-6.

96. Hsu AJ, Carson KA, Yung R, Pham PA. Severe prolonged sedation associated with coadministration of protease inhibitors and intravenous midazolam during bronchoscopy. Pharmacotherapy. 2012;32(6):538-45.

97. Greenblatt DJ, von Moltke LL, Harmatz JS, Durol AL, Daily JP, Graf JA, et al. Differential impairment of triazolam and zolpidem clearance by ritonavir. J Acquir Immune Defic Syndr. 2000;24(2):129-36.

98. Culm-Merdek KE, von Moltke LL, Gan L, Horan KA, Reynolds $\mathrm{R}$, Harmatz JS, et al. Effect of extended exposure to grapefruit juice on cytochrome P450 3A activity in humans: comparison with ritonavir. Clin Pharmacol Ther. 2006;79(3):243-54.

99. Bandera R, Bollini P, Garattini S. Long-acting and short-acting benzodiazepines in the elderly: kinetic differences and clinical relevance. Curr Med Res Opin. 1984;8(Suppl 4):94-107.

100. Cao B, Wang Y, Wen D, Liu W, Wang J, Fan G, et al. A trial of lopinavir-ritonavir in adults hospitalized with severe Covid-19. N Engl J Med. 2020;382(19):1787-99.

101. Chen J, Xia L, Liu L, Xu Q, Ling Y, Huang D, et al. Antiviral activity and safety of darunavir/cobicistat for the treatment of COVID-19. Open Forum Infect Dis. 2020;7(7):ofaa241.

102. Anmella G, Arbelo N, Fico G, Murru A, Llach CD, Madero S, et al. COVID-19 inpatients with psychiatric disorders: Realworld clinical recommendations from an expert team in consultation-liaison psychiatry. J Affect Disord. 2020;274:1062-7.

103. Jorgensen SCJ, Kebriaei R, Dresser LD. Remdesivir: review of pharmacology, pre-clinical data, and emerging clinical experience for COVID-19. Pharmacotherapy. 2020;40(7):659-71.

104. Shen Y, Eades W, Yan B. Remdesivir potently inhibits carboxylesterase-2 through covalent modifications: signifying strong drug-drug interactions. Fundam Clin Pharmacol. 2020. https:// doi.org/10.1111/fcp.12643 (epub ahead of print). 